\title{
Toward a clinical optoretinogram: a review of noninvasive, optical tests of retinal neural function
}

\author{
Ravi S. Jonnal^ \\ Department of Ophthalmology and Vision Science, University of California, Davis, CA, USA \\ Correspondence to: Ravi S. Jonnal. Department of Ophthalmology and Vision Science, University of California, Davis, CA, USA. \\ Email: rjonnal@gmail.com.
}

\begin{abstract}
The past few years have witnessed rapid development of the optoretinogram—a noninvasive, optical measurement of neural function in the retina, and especially the photoreceptors (Ph). While its recent development has been rapid, it represents the culmination of hundreds of experiments spanning decades. Early work showed measurable and reproducible changes in the optical properties of retinal explants and suspensions of $\mathrm{Ph}$, and uncovered some of the biophysical and biochemical mechanisms underlying them. That work thus provided critical motivation for more recent work based on clinical imaging platforms, whose eventual goal is the improvement of ophthalmic care and streamlining the discovery of novel therapeutics. The first part of this review consists of a selective summary of the early work, and identifies four kinds of stimulus-evoked optical signals that have emerged from it: changes in light scattered from the membranous discs of the Ph's outer segment (OS), changes in light scattered by the front and back boundaries of the OS, rearrangement of scattering material in and near the OS, and changes in the OS length. In the past decade, all four of these signals have continued to be investigated using imaging systems already used in the clinic or intended for clinical and translational use. The second part of this review discusses these imaging modalities, their potential to detect and quantify the signals of interest, and other factors influencing their translational promise. Particular attention is paid to phase-sensitive optical coherence tomography (OCT) with adaptive optics (AO), a method in which both the amplitude and the phase of light reflected from individual $\mathrm{Ph}$ is monitored as visible stimuli are delivered to them. The record of the light's phase is decoded to reveal a reproducible pattern of deformation in the OS, while the amplitude reveals changes in scattering and structural rearrangements. The method has been demonstrated in a few labs and has been used to measure responses from both rods and cones. With the ability to detect responses to stimuli isomerizing less than $0.01 \%$ of photopigment, this technique may prove to be a quick, noninvasive, and objective way to measure subtle disease-related dysfunction at the cellular level, and thus to provide an entirely new and complementary biomarker for retinal disease and recovery.
\end{abstract}

Keywords: Optoretinography (ORG); adaptive optics (AO); optical coherence tomography (OCT); functional imaging; photoreceptors $(\mathrm{Ph})$

Submitted Sep 18, 2020. Accepted for publication Jul 12, 2021.

doi: $10.21037 /$ atm-20-6440

View this article at: https://dx.doi.org/10.21037/atm-20-6440

\footnotetext{
$\wedge$ ORCID: 0000-0002-9545-1837.
} 


\section{Introduction}

Human vision begins in the retina, when light is converted by photoreceptors $(\mathrm{Ph})$ into a "substantial chemical image" (1), which is then post-processed by retinal interneurons before transmission to the brain by retinal ganglion cells. The most significant impact of retinal diseases and ocular manifestations of systemic disease is loss of vision, ultimately caused by dysfunction and/or demise of retinal neurons. At present, the mainstays of functional neural testing in the retina are psychophysical (such as acuity testing, visual fields, and microperimetry) and electrophysiological [such as the electroretinogram (ERG), multifocal electroretinogram (mfERG), and visual evoked potential (VEP)]. These methods have been used to learn much about disease-related neural dysfunction, especially when they have been used to localize dysfunction to individual classes of neuron and have permitted correlation with structural changes visible in clinical imaging. The use of these methods in clinical research and practice have been reviewed in detail elsewhere (2-5).

Clinical psychophysics and electrophysiology are fundamentally blunt methods, since they measure the bulk subjective and electrical responses of the retina, respectively. Nevertheless, investigators in these fields have developed countless ways to tune visible stimuli and analyze responses in order to improve the specificity and resolution of the tests. Psychophysical tests can routinely distinguish the responses of cones and rods, and differentiate the responses of $\mathrm{Ph}$ from those of postreceptoral neurons. The fullfield ERG, through the use of adapting backgrounds, can similarly distinguish the signals of rods and cones, and through temporal analysis of the bulk electrical response of the retina can separate the electrical responses of $\mathrm{Ph}$, bipolar cells, ganglion cells, and cells of the retinal pigmented epithelium (RPE). Perimetry and the multifocal (mf) ERG , moreover, permit spatial localization of responses as well, although with resolution much coarser than that offered by adaptive optics (AO). AO perimetry permits diffractionlimited stimulus delivery and imaging $(6,7)$, and thus offers the possibility of psychophysical testing with cellular resolution. In spite of these developments, researchers have for decades investigated possible optical signatures of neural function in the retina, motivated by the hope for rapid, noninvasive, and objective methods that might provide additional, complementary information about retinal function and dysfunction.
The past three decades have witnessed a revolution in our ability to resolve structures in the retina at cellular scales (Figure 1). First, optical coherence tomography (OCT) provided the first cross-sectional images of the retina (8), and developments in dispersion control over broad bandwidths (9) armed OCT with the axial resolution sufficient for differentiating the lamina of the neural retina (10) (Figure 1B). Soon after, spectral-domain (SD) (or Fourier-domain) OCT was developed (11-14), which further improved axial resolution, speed, and sensitivity of OCT. Meanwhile, other investigators were discovering how to overcome the fundamental limitations on lateral resolution in the living human eye, namely by using AO to compensate for the dominant effect of dynamic aberrations when imaging through a dilated pupil (15). The axial and lateral resolutions of these two technologies were combined in AO-OCT (16-18), which has been used to image the retina with microscopic three-dimensional resolution and perform quantitative morphology at the cellular scale (19-21). As our abilities to resolve retinal neurons axially and laterally (first separately and later together) have grown, so too has the interest in using these technologies to detect and measure their functional responses.

In this review, I use the term optoretinography (ORG) to mean "measurement of light-evoked changes in the optical properties of retinal neurons". As we shall see, this is a sufficiently broad definition to include dozens of methodological and analytical variants. However, it is narrow as well, excluding light-evoked changes in blood flow or other spectroscopic parameters. I would like to avoid the broader term "functional", which includes the latter (22), in order to make the disciplinary boundaries clear. The term "optoretinogram" was first used aspirationally by investigators who had hoped to identify light-evoked optical responses in vivo (23). More recently it has been used as an umbrella term for optical measurements of retinal neural function (24).

Because OCT and AO technology has developed so rapidly, we are now able to observe the retina in great detail, in vivo (Figure 1). In some cases, these in vivo observations have outpaced our understanding of the underlying anatomical structures (25-29). Given that any attempt to understand the structure of the retina is susceptible to methodological artifacts, it is not clear what the "gold standard" should be, even for structural imaging. The addition of functional responses to this milieu multiplies the problem. If ORG measurements are to be interpreted 


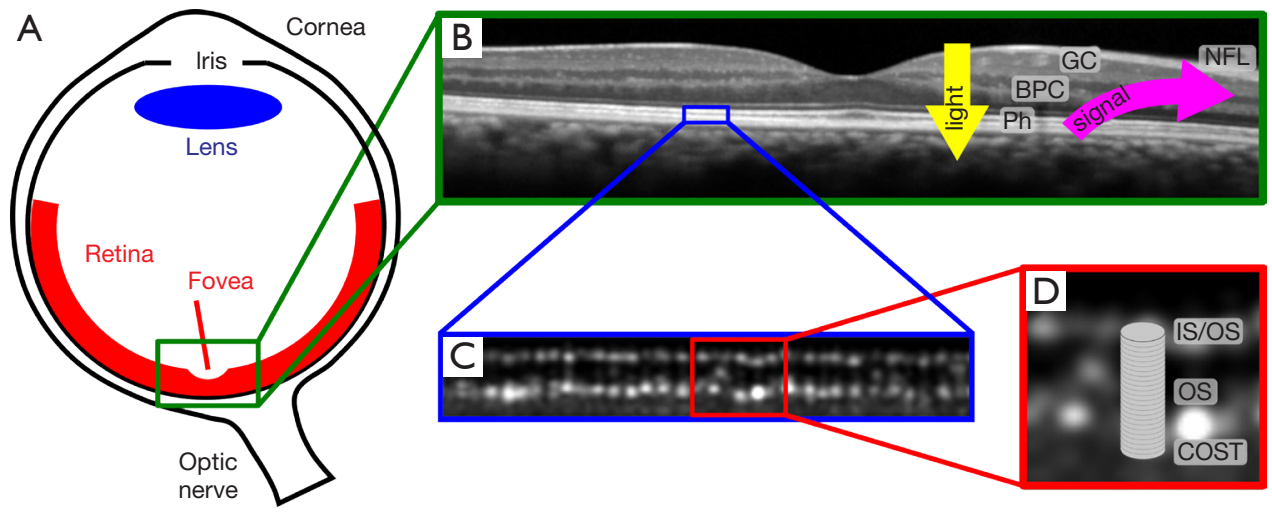

Figure 1 Overview of retinal anatomy, phototransduction, and retinal imaging using OCT and AO-OCT. (A) A cross-sectional diagram of the eye, as viewed from above. An image is formed on the retina when the eye's two main optical elements-the cornea and lens- focus the incident light, while the iris controls the amount of light entering the eye. The center of the retina, called the fovea, is specialized for high acuity vision, and is thus densely packed with cone $\mathrm{Ph}$. (B) Optical coherence tomography (OCT) is a standard of ophthalmic care, providing clinicians with a cross-sectional image of the retina. Shown here is a scan through the macular region, including the fovea and foveal pit. Clinical OCT systems have axial resolution on the order of $10 \mu \mathrm{m}$, sufficient for resolving the layers of the neural retina. Light incident on the retina must penetrate the inner neural layers into the outer retina, where the $\mathrm{Ph}$ lie. The $\mathrm{Ph}$ send signals to the BPC, which in turn signal the GC, whose axons form bundles in the NFL. These bundles meet to form the optic nerve, and carry the retinal signal to the brain. While the main neural layers are resolved in clinical OCT images, lateral resolution is insufficient to individuate cells. (C) A cross-sectional image acquired with AO-OCT. AO provides the lateral resolution required to observe single cells and subcellular features. This AO-OCT image shows two correlated arrays of scattering objects, believed to be the inner and outer boundaries of the photoreceptor OS. (D) These boundaries are called the IS/OS and COST or ROST. The OS consists of a cylindrical, membrane-bound stack of between 200 and 1,000 circular membranous discs, studded with light-sensitive proteins called opsins. The capture of photons by opsins in the OS is the first step in seeing. OCT, optical coherence tomography; AO, adaptive optics; Ph, photoreceptors; BPC, bipolar cells; GC, ganglion cells; NFL, nerve fiber layer; OS, outer segments; IS/OS, inner segment outer segment junction; COST, cone outer segment tips; ROST, rod outer segment tips.

in the context of what is known about retinal anatomy and neurophysiology, much more effort should be put toward understanding both the anatomical structures and the biophysical mechanisms where they originate.

\section{Optical signatures of signal transduction in neurons, or intrinsic signals}

When neurons transduce or conduct signals, many biochemical and biophysical changes occur: the movement of ions across the membrane changes, along with attendant water movement; distribution of charge about the membrane changes; the involvement of proteins (e.g., signal transduction catalysts and ion channels), often resulting in conformational changes in distribution of mass and/ or charge or dissociation of subunits. For eighty years scientists have studied the potential optical consequences of these processes (30). Such effects were first observed in the walking leg nerve bundle of the green crab, by David Keynes Hill and Richard Darwin Keynes in 1949 (31). They found that scattering of white light increased in response to stimulation of the nerves, and initially hypothesized that this was due either to the scattering properties of the axoplasm or the membrane. Subsequent experiments, by Hill, on an analogous nerve bundle of the spider crab suggested that the scattering changes may be biphasic, with an initial increase followed by a decrease (32). Those experiments also revealed that the addition of sodium chloride or magnesium sulfate to the extracellular medium, known to cause osmotic shrinkage of the axons in the bundle, caused a concomitant increase in scattering by the bundle. This finding suggested that the initial hypotheses were incorrect, and that the scattering increase was more likely due to changes in the spatial distribution of the axons within the surrounding extracellular medium. Hill stated that the causal relationship between axon volume and scattering was unknown, but 
nevertheless attempted to calculate the former from the latter, a calculation he admitted "has necessarily to be rather inaccurate". The process by which changes in tissue manifest as changes in scattering is complex, and implicates many optical, biochemical, and biophysical factors. The optical processes themselves are numerous, and include those dependent on material refractive index (such as refraction and scattering) and those not (such as diffraction). In the same issue, Hill published results of a separate experiment which showed that stimulation of a single cuttlefish axon produced an initial decrease, followed by an increase, in both the axon's diameter and length (33). Since then, morphological changes have been measured in dissected neurons using a variety of imaging techniques, such as scattering (34-36), fluorescence and polarization imaging (37), laser interferometry (38), atomic force microscopy (39), optical levers (40), OCT $(41,42)$, phasesensitive reflectometry (43), and phase-sensitive OCT (44).

Researchers studying the physiology of $\mathrm{Ph}$ soon became interested in the effects of stimulation on their optical properties. Korenbrot and Cone showed light-dependent resistance to sodium influx in isolated frog rods, using an imaging cytometry system (45). Bownds and Brody employed an early impedence cytometer (or Coulter counter) to measure the volume of isolated bullfrog rod outer segments (OS) (46). They found that in the dark, in a hypotonic suspension, the rod OS swelled, as predicted, but that this swelling response could be attenuated or eliminated by light exposure. These experiments provided important confirmation for the theory that light-evoked hyperpolarization of $\mathrm{Ph}$ was affected by closure of sodium channels, but also blazed a trail for investigators looking for optical signatures of $\mathrm{Ph}$ signaling. The observation that hypotonic swelling was eliminated by light exposure supports the idea that in physiological conditions light exposure affects the volume of the $\mathrm{Ph}$ OS.

Corless showed a difference in the $\mathrm{X}$-ray diffraction pattern resulting from dark-adapted and fully bleached frog rods (47). A year later, Chabre and Cavaggioni devised a system for measuring similar diffraction patterns dynamically, and showed that in retinal explants from frogs, upon stimulation with white light, the rod OSs initially shorten by $0.5 \%$, and then elongate by $2 \%$ (48). The authors reported an initial broadening of the diffraction peaks, suggesting disorganization of the OS lattice. They attributed the disorganization of the discs to the intensity of the stimulus flash, which bleached all of the photopigment within a few seconds, and noted that this condition does not arise naturally. In a later experiment, the same investigators observed that the second, swelling phase was only present in retinal explants, and not in single isolated frog rods (49).

Since efforts to measure light-evoked changes in the $\mathrm{Ph}$ OS length have typically observed both increases and decreases in OS length, "deformation" will be used in this manuscript as an umbrella term for changes in OS length. The mechanisms involved in deformation may include increases or decreases in the disc spacing or volume with concomitant changes in the bulk length of the OS.

As $\mathrm{X}$-ray imaging of $\mathrm{Ph}$ was not possible in vivo, researchers began to consider how OS deformation may affect the light scattering properties of the $\mathrm{Ph}$. This is analogous to the early work of Hill and Keynes, which showed that axonal swelling manifested as scattering changes in bundles of axons. McConnell showed that when suspensions of bovine rod OSs were exposed to light, their scattering properties were affected, with the sign and magnitude of scattering changes dependent on concentrations of salts in the medium (50).

$\mathrm{Ph}$ are rich with photopigment-the molecules responsible for absorbing light and initiating the phototransduction cascade. Photopigments complicate functional imaging because their scattering properties change with activation (bleaching), independent of downstream signaling. A number of investigators began to study light-evoked scattering of near-infrared (NIR) light instead, mainly in suspensions of frog and bovine rod OSs or rod OS discs. These investigators characterized both gross changes in scattering and angular changes in scattering. For the sake of simplicity here only increases and decreases in scattering are described. For heterogeneous suspensions, this is sufficient, as changes in angular profile are difficult to interpret when a variety of particle sizes are present. "Increased scattering" here is equivalent to decreased transmission through the suspension, as measured $180^{\circ}$ away from the source. In the case of NIR light, which is known to have low absorbance in the $\mathrm{Ph}$, a decrease in transmission implies an increase in scattering in other directions, possibly isotropic.

Hofmann et al. determined that there were multiple mechanisms for light-evoked scattering changes, each with different kinetics. A decrease in scattering was attributed to swelling of the whole rod OS, with overlapping increases in scattering due to shrinking of the discs (51). A year later, the same group was able to isolate the scattering increasethe positive ("P") signal-in suspensions of bovine rod OS discs with their plasma membranes broken (52). They 
attributed this increase in scattering to a contraction of the discs, and determined that it is proportional to bleaching at fractions less than $2 \%$ and saturates when $15 \%$ of rhodopsin is bleached. Another experiment showed that toad rods exhibit an initial increase in scattering followed by a slower decrease (53). The investigators attributed these changes to shrinking and swelling, respectively, of the OS discs, confirming the earlier work. In parallel, other investigators found that light stimuli affected the transverse birefringence of frog rod OSs (54). Disc spacing affects polarizationdependent phase retardation, as shown by diurnal variations Nomraski images (55).

Scattering changes-and the putative underlying changes in disc volume-were later suggested to be caused by binding and dissociation of activated rhodopsin $\left(\mathrm{R}^{*}\right)$ with its GTPbinding protein, transducin, and associated changes in the mass of the disc membrane (56-58). Kühn et al. developed a method for removing transducin from rod OS discs and then titrating it back onto the disc membrane (56). They distinguished separate scattering changes, for the respective binding (P-signal) and dissociation ( $\mathrm{N}$-signal) of $\mathrm{R}^{*}$ and transducin, by making measurements while modulating the concentration of GTP, which is required for phosphorylation and subsequent dissociation of transducin's $\alpha$-subunit. Hofmann et al. found that the saturation of the P signal occurred at approximately $10 \%$ of rhodopsin bleaching (similar to the $15 \%$ reported by Uhl et al.), and was shown to be modulated by removal or addition of transducin to the discs (57). These findings, along with the 10:1 ratio of rhodopsin to transducin in the bovine rod (59), provide evidence that the light-evoked increase in scattering ( $\mathrm{P}$ signal) originates from the binding of $\mathrm{R}^{*}$ and transducin.

Using magnetically oriented frog rod OSs, Vuong et al., separated the contributions to scattering changes that were due to axial and transverse light paths through the OS (60). They determined that a portion of the previously reported light-induced reductions in scattering by suspensions of rod OS were due to light passing transversely through the OS, while the light passing axially through the OS experienced increased scattering due to visible stimuli. A few years later, Vuong et al. investigated the impacts of light stimulation on diffraction of neutron beams by the rod OS (61). Observed shifts in the diffraction pattern peaks confirmed the earlier work by Chabre and Cavaggioni, that light stimuli reduce the lattice spacing in the rod OS by about $0.3 \%$. They also observed that the intensity of the diffraction peaks increased, with faster kinetics than the lattice change. They attributed the intensity changes to rearrangements of mass about the disc membrane, in particular to movement of the GTP-bound transducin $\alpha$ subunit away from it. The lattice change, they argued, was a slower osmotic readjustment of the rod OS.

In addition to $\mathrm{R}^{*}$-transducin interactions, transducinphosphodiesterase (PDE) interactions were shown to generate a distinct scattering signal. This amplified, transient (AT) signal was demonstrated in suspensions of bovine rod OSs $(62,63)$ and bovine retinal explants (64). Pepperberg et al. showed that the scattering changes were present in low $\mathrm{Na}^{+}$solutions, even when the ERG response was completely extinguished (64), thereby ruling out the possibility that the former were due to the shifting $\mathrm{Na}^{+}$ gradient underlying membrane hyperpolarization.

A thorough description of the literature on the transducin binding, dissociation, and AT signals is out of the scope of this review, but these observations suggest that several steps in the phototransduction cascade cause scattering changes with different signs and magnitudes. Arshavsky, Lamb, and Pugh present a more detailed review (65), and argue that in samples that retain the most natural structure, such as explants in physiological perfusions, the sum of the scattering changes has a simplified presentation, with a light-evoked increase in non-forward scattering (including backscattering). This suggestion offered a way to reconcile apparently inconsistent findings, and supported the idea that light-evoked scatter in the $\mathrm{Ph}$ could be used to assess their health objectively and noninvasively.

\section{From microscope to ophthalmoscope}

The rest of this review will focus on NIR imaging of lightevoked changes in the living eye, using methods that have translational promise. In parallel to the latter efforts, many investigators have continued work on animal models. Much of this work has been recently reviewed by Hunter et al. (66) and Yao and Kim (67). Hunter et al. as well as Miller and Kurokawa (21) include reviews and additional perspectives on the human work described below. The goal of this review is to describe the state of the field of non-invasive, NIR imaging of stimulus-evoked activity in retinal neurons. Intentionally omitted from this review are several other approaches for measuring physiological changes in $\mathrm{Ph}$, namely densitometry (68), measurement of OS renewal (69) and disc shedding (70), functional oximetry (71), functional angiography (72), and other studies of changes of unknown origin $(73,74)$.

The findings summarized above have been collectively 
described as intrinsic signals, in concert with parallel efforts to identify activity-dependent changes in the optical properties of cortical neurons. In light of the diversity of experiments conducted, however, it is useful to identify two methodological categories suitable for in vivo human imaging: (I) those which measure changes in the scattering properties of the $\mathrm{Ph} \mathrm{OS}$, whether arising from the discs, plasma membrane, or both; (II) those which measure changes in the volume of the OS. As investigators began to measure human $\mathrm{Ph}$ responses, initially most efforts focused on the first, but much progress on the second has been made in recent years. Note that these are methodological categories; the mechanisms underlying the observed signals may overlap or be identical.

\section{Reflectometry and ophthalmoscopy}

The first effort to measure light-evoked scattering changes in the living human retina was based on singlespot densitometry, which utilized a wheel of interference filters to measure reflectance changes at twelve discrete bands between 560 and $740 \mathrm{~nm}$ during light and dark adaptation (75). Subjects were dark-adapted for 30 minutes, followed by 15 minutes of measurement in the dark (baseline), 16 minutes in the light, and 35 minutes in the dark again. Across the visible spectrum, reflected light changed in ways that were roughly consistent with earlier densitometric measurements of photopigment bleaching and recovery-namely increasing in response to photopigment bleaching, and returning to baseline during the first few minutes of the ensuing dark. However, they also observed a slow, constant reduction in reflectance during the 16-minute light-on period, inconsistent with predictions of steadystate bleaching. In contrast, in the NIR $(740 \mathrm{~nm})$, exposure to visible light caused only a decrease in reflectance, and turning off the light caused a further decrease in reflectance (for a few minutes) followed by an increase to baseline. The investigators found that the pattern of change, across the measurement spectrum, was consistent with a twofactor model, including a component due to photopigment bleaching and another, which they attributed to changes in refractive index of either OS discs or extracellular matrix (ECM). By analyzing the dependence of the second factor on stimulus wavelength and the position of the beam in the pupil, they provided confirmation that the observed changes originated in the $\mathrm{Ph}$.

In 2004, Tsunoda et al. measured light-evoked reductions in NIR backscattering in anesthetized macaques (76). The largest changes were observed in the macula. The amplitude and spatial distribution of the reductions were highly correlated with the those of mfERG responses. Soon after, Abràmoff $e t$ al. used a clinical fundus camera with a $780 \mathrm{~nm}$ illumination source to measure fundus reflectance changes following delivery of green visible stimuli (77). They found a reduction in fundus reflectance of $0.14 \%$ following onset of the $3 \mathrm{~s}$ stimulus, followed by a slow recovery after light offset.

Despite methodological differences, DeLint et al., Tsunoda et al., and Abràmoff all observed light-evoked reductions in retinal NIR reflectance, probably due to $\mathrm{Ph}$, with light-onset causing a reduction in scattering. Nevertheless, these observations are inconsistent with those made in ex vivo preparations of OS fragments, and especially inconsistent with those described by Arshavsky et al. (65) as retaining natural structure, e.g., Vuong et al.'s magnetically aligned OSs (60). The reasons for this inconsistency remain unknown.

\section{Early AOs ophthalmoscopy}

Between 1997 and 2002, investigators had successfully combined AO with ophthalmoscopic modalities, namely flood illumintion (AO-FI) fundus imaging (15) and scanning light ophthalmoscopy (AO-SLO) (78) to obtain images of the living human retina with cellular resolution. These approaches initially permitted resolution of human cones, and many efforts were soon underway to characterize their anatomical and physiological characteristics. Among these were several that attempted to measure light-evoked changes in the retina.

Our group used an AO-FI system with coherent illumination and a $192 \mathrm{~Hz}$ frame rate to demonstrate that the reflectance of human cones oscillates with a chirped sinusoidal pattern after stimulus flashes (79). We showed that the observed responses were dependent on the coherence length of the light source. This was done by reducing the coherence length and showing a resulting reduction in the number of cones exhibiting a response with a clear reduction in the amplitude of the responses.

When light from reflective objects at different optical path lengths (OPLs) (or depths in the retina) interferes, the amplitude of detected signal depends on the path length difference and the coherence length of the light source [the distance over which the light's phase is sufficiently correlated to generate an interference pattern (Figure 2)]. Our observations implied that the source of 


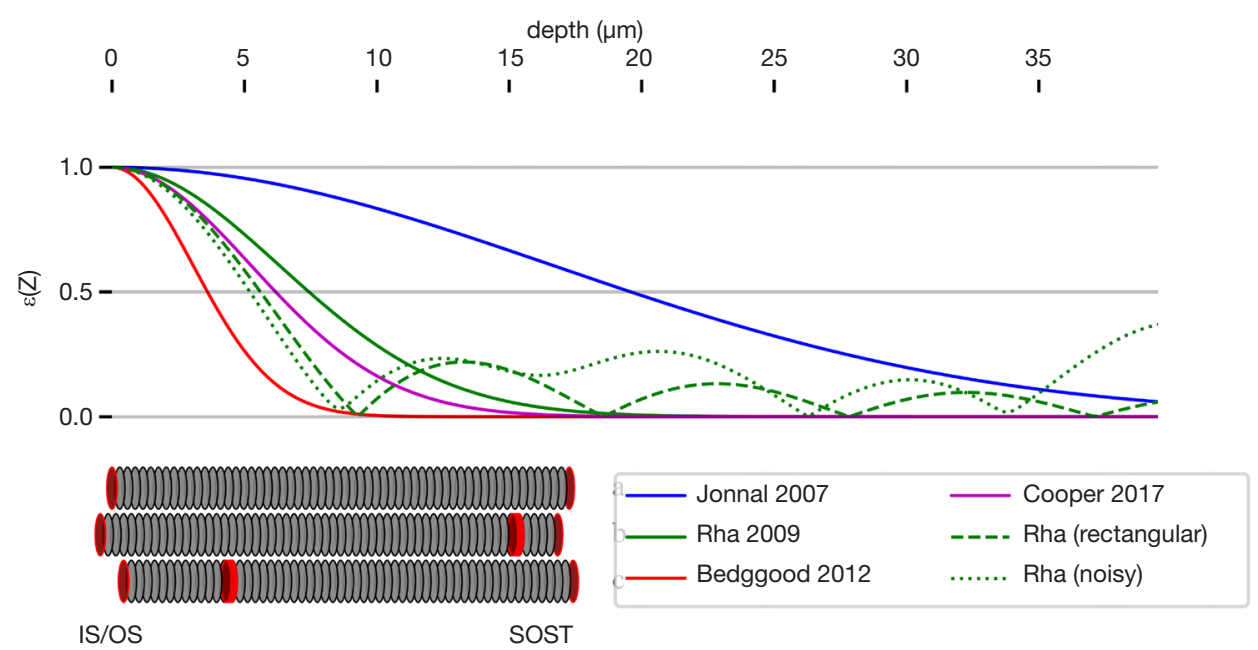

Figure 2 Hypothetical origins of light-evoked changes in coherent flood illumination and SLO images of cones. Jonnal et al., 2007 reported the first observations of stimulus-evoked changes in the intensity of areal images of the cone $\mathrm{Ph}$, using an adaptive optics flood illumination camera (79). They showed that the resulting oscillations in cone reflectance were dependent on the coherence of the illumination source, and hypothetically attributed them to elongation of the cone OS in the presence of coherent interference between light reflected by its two ends, the IS/OS and COST. The temporal coherence function $[\varepsilon(\mathrm{z})]$ of a source describes how the phase of a light beam decorrelates as it propagates through space and, thus, how the amplitude of an interference fringe falls as the interfering scatterers are axially displaced. The coherence function is usually summarized by its FWHM. After Jonnal et al., 2007, several investigators (80-82) reported similar oscillations in cone reflectance, using sources with coherence lengths shorter than the OS length of the cones. Two hypothetical explanations for the are shown above. At the top are plots of coherence functions based on several reported bandwidths or coherence lengths (solid lines). At the bottom are three cartoon cone outer segments, showing the primary reflectors at IS/OS and COST, as well as additional OS reflections observed in many cones. The coherence functions plotted with solid lines are Gaussian functions with width parameters derived from the reported FWHM. FWHM is a convenient measure of source coherence because the source spectrum and coherence functions have a simple relationship when they are Gaussian. In practice, however, neither spectra nor coherence functions are Gaussian. The dotted and dashed lines show the coherence functions that would result if Rha et al.'s (80) spectrum were rectangular in shape or Gaussian with a small amount of noise, respectively, but with the same spectral FWHM. Departures from a Gaussian shape can have significant effects in the tails of the coherence function, and could lead to high fringe amplitude even when none is predicted. Complicating matters further, as reported by Azimipour et al., 2019, the photoreceptor OS often has additional reflections (depicted in red in the cartoons), observed to move and change amplitude upon stimulation. These additional reflections are likely to contribute to the en face measurement, significantly complicating quantification even when coherence is shorter than the OS. SLO, scanning light ophthalmoscopy; Ph, photoreceptors; OS, outer segments; IS/OS, inner segment outer segment junction; COST, cone outer segment tips; FWHM, full-width at half maximum.

these oscillations was coherent interference between two reflectors. Since the phase of the oscillations was correlated within cones and uncorrelated among cones, we inferred that the reflective surfaces originated within the cones. We hypothetically attributed the oscillations to interference between reflections from the $\mathrm{Ph}$ inner-outer segment junction (IS/OS) and the cone OS tips (COST), combined with a change in the OS OPL. The frequency and number of cycles of oscillation increased with stimulus dose, suggesting that the hypothetical elongation was a side effect of the phototransduction process.
Around the same time, Grieve and Roorda demonstrated light-evoked changes in scattering from $\mathrm{Ph}$ using an AO-SLO (83). They observed in some subjects that the reflectance of cones, when averaged over many cones, increased by $5 \%$ in response to a constant $3 \mathrm{~s}$ stimulus, or $1-2 \%$ in response to $2 \mathrm{~s}$ of $15 \mathrm{~Hz}$ flicker. Since their imaging source had a coherence length of about $5 \mu \mathrm{m}$ substantially shorter than the cone OS in the macula, they did not attribute any of the responses to interference between the IS/OS and COST. The amplitude of the observed changes-a few percent-is consistent with 
observations of changes in scattering from the discs.

Over the ensuing decade, several investigators produced results that appeared to refute the hypothesis that the observed oscillations in cone reflectance were due to interference between light scattered from IS/OS and COST. In particular, Rha et al. (80) and Bedggood and Metha (81) showed stimulus-evoked oscillations in cone reflectance with illumination sources that had theoretical coherence lengths considerably shorter than the imaged cone's OSs. The roles of cellular structure and illumination coherence length in generating an interference signal are illustrated in (Figure 2).

Rha et al. used a custom AO-FI system with a partially coherent $840 \mathrm{~nm}$ source to observe oscillations in human cones in response to visible flashes. Their light source had a coherence length of $15 \mu \mathrm{m}$, shorter than the OS of the cones they imaged at $1-2^{\circ}$. Nonetheless, they observed changes in a majority of cones. These changes were categorized as oscillating, increasing, or decreasing; in the cases of the latter two, the amplitudes of the observed changes $(>10 \%)$ were much higher than those reported by Grieve et al., and similar to those reported by our group (10-30\%). The implication of these relatively large amplitudes is that the observed effects were caused by interference, and not due to the smaller changes attributed to scattering from the discs.

In 2012, Bedggood and Metha used AO-FI with a single $\sim 560 \mathrm{~nm}$ source to image and stimulate human $\mathrm{Ph}$, and observed predicted exponential increases in cone reflectance due to optical density changes associated with bleaching (81). The source's coherence length was $\sim 7 \mu \mathrm{m}$, but they nevertheless observed a number of cones exhibiting intensity oscillations consistent with interference. Soon after, using the same visible source with a substantially faster, $1 \mathrm{kHz}$ camera, they were able to reproduce the same observations of reflectance oscillation, while also showing that the onset of the oscillations occurred within $2 \mathrm{~ms}$ of stimulus onset. This provided stronger evidence that the optical changes begin during phototransduction in the cones. Cooper et al. later showed a similar result using an AO-SLO with an infrared source with a $15 \mu \mathrm{m}$ coherence length, and also proposed a repeatable way to quantify these responses by integrating the signal over hundreds of cones (82).

All of the early AO ophthalmoscopic experiments used sources capable of generating some interference in the OS, and thus we cannot rule out the possibility that the underlying mechanism was movement of scatterers combined with common path interference, as opposed to changes in the cells' scattering properties. At the time, axial localization of the light-evoked effects hadn't been established in single cells; recent evidence of light-evoked movements of subcellular scatterers makes coherent interference an even more plausible explanation for these observations see (Figure 2).

\section{OCT}

\section{OCT measurements of light-evoked changes in scattering}

While investigators were measuring scattering changes in the human retina with reflectometry and fundus imaging, OCT was emerging as a standard of clinical ophthalmic care and researchers began to investigate whether it could be used to reveal the same changes. Unlike fundus imaging, densitometry, and SLO, OCT offered the promise of axial localization of the scattering changes and associated improved signal-to-noise ratio (SNR) for light-evoked scatter. Initial experiments were conducted on animal models. First, Yao et al. used a time-domain (TD) OCT system to measure light-evoked changes in the frog retina (84). They leveraged an electro-optic phase modulator to facilitate high-speed scanning, and achieved an axial resolution of $19 \mu \mathrm{m}$. After stimulation of darkadapted retinal explants, they measured a decrease in scattering following a $10 \mathrm{~ms}$ stimulus flash, which returned to baseline in about one second.

Soon after, Bizheva et al. developed a broader band TD-OCT system, with a resulting axial resolution of $<3 \mu \mathrm{m}(85)$. They used this system to measure lightevoked scattering changes in isolated rabbit retina. The exquisite detail of the retinal layers visible in these images and contemporaneous SD OCT images was new, and the nomenclature for the retinal layers was in flux. Investigators found changes in two layers now referred to as the IS/OS and RPE bands $(86,87)$, although we now believe that the latter contains contributions from the OS tips as well. In the IS/OS and RPE bands, the stimulus flash was found respectively to decrease by $20 \%$ and increase by $70 \%$ the amplitudes of backscattered light. Although the authors did not describe additional $\mathrm{Ph}$ changes in detail, other clear changes were visible within the OS, namely a reduction in signal at its proximal portion and an increase in signal at its distal portion. In the RPE band, some axial movement or segregation of signal was evident.

In the same year, Srinivasan et al. measured similar changes in the living rat retina, enabled by the higher speed 
and sensitivity of spectral domain (SD)-OCT (88). They observed that when the rat was dark-adapted, the OCT signal-integrated over a $10 \mu \mathrm{m}$ axial zone corresponding to the OS-increased in response to light stimuli by about $12 \%$. This increase was smaller by a factor of six in lightadapted animals. While there is an apparent inconsistency between the measurements of Bizheva et al. and Srinivasan et al., it may be resolved by observing that the former measured changes at the cellular interfaces and the latter measured changes within the OS. Also, while rabbit and rat retinae are similar, with large majorities of rods closely apposed to the RPE apical surface, we cannot rule out the possibility of interspecific differences in their optical responses to light. Given the difficulty of knowing precisely the shape of the axial point spread function (PSF) in tissue, it is possible that the changes measured in the OS originated at its boundaries and were blurred into the OS by the system's axial PSF.

These experiments on rabbit and rat models paved the way for the first OCT measurement of light-evoked changes in the human retina (89). Srinivasan et al. used an SD-OCT system to show that stimulation with visible light increases backscattering from the IS/OS and decreases backscattering from the rod OS tips (ROST), which are very close to the RPE. The magnitude of responses varied with retinal eccentricity, but were between $3-5 \%$. This was a paradoxical finding, when compared with Bizheva et al. since the measured changes in analogous layers of isolated rabbit retinae had opposite signs. Bizheva et al.'s result was consistent with a reduction in OS refractive index, e.g., due to water moving into the OS, while Srinivasan et al.'s result suggests the opposite.

The next year, Schmoll et al. did in vivo measurement of light-evoked changes in human $\mathrm{Ph}$ using a custom SDOCT system. They showed that by flickering the stimulus at $5 \mathrm{~Hz}$ and band pass filtering the resulting scans, they could improve the SNR of this signal (90). They showed light-evoked increases in backscattering from the IS/OS and COST, of about $3 \%$ and $2 \%$, respectively. The finding at the IS/OS was consistent with Srinivasan et al. (89).

Based on earlier work done in the mouse (91), Bissig et al. studied how Alzheimer's disease affects light-evoked changes in the retinal OCT (92). To establish a baseline effect, they collected clinical OCT scans on young, healthy volunteers after dark adaptation and again after exposure to a bright white LED, just beside the fovea. They found that light backscattered from IS/OS and COST increased between $3 \%$ and $7 \%$, within a few seconds of the bleaching exposure. In the Alzheimer's patients, the light-evoked changes in Ph effects were normal, but abnormalities in the response were observed in other retinal layers.

Several of the experiments above showed stimulusevoked increases in backscattering from IS/OS, which is consistent with an increase in the refractive index mismatch between the inner segments (ISs) and OSs. Since the lipiddense OS is thought to have a higher baseline refractive index than the IS, these results suggest that visible stimuli increase the OS refractive index. Such an increase could be due to water movement out of the OS. Nevertheless, numerous inconsistencies exist among the findings, including some paradoxical observations of decreased IS/ OS backscatter. The reason for these inconsistencies may lie among the many differences between the experimentsimaging wavelengths, speed, stimulus protocols, or interspecific differences in the refractive indices of the IS and OS. However, it is also worth noting that while refractive index mismatch is a relatively simple and accurate model for OCT reflections, the ultrastructure of the IS/ OS and OS tips is complicated, and AO-OCT studies have shown that the IS/OS it may have finite axial extents, on the order of $1-3 \mu \mathrm{m}(26,29)$.

\section{OCT measurements of light-evoked changes in OS length}

In parallel to measurements of changes in backscattering from retinal surfaces, other investigators were studying changes in the positions of the scattering surfaces, IS/OS and the OS tips in particular. Stimulus-evoked changes in the positions of either one implies changes in the length of the OS. Abràmoff et al. were the first to use OCT to measure light-evoked changes in the length of the cone OS (93). They used a clinical SD-OCT system to monitor the OS effective length in 11 subjects without known retinal disease, and found that this distance reduces by 1-3 $\mu \mathrm{m}$ during the first ten minutes of adaptation to light. While clinical OCT cannot reliably distinguish between COST and the RPE/ROST complex everywhere in the retina, these bands can be distinguished in some subjects sufficiently to measure light-evoked changes.

Zhang et al. measured light-evoked changes in the axial backscattering profile of the rod-dominant mouse retina (94), using a custom SD-OCT system with an axial resolution of $2 \mu \mathrm{m}$ (95). They showed that a light flash that bleaches $10 \%$ of rhodopsin increases backscattering from the IS/OS and ROST, but the changes measured were substantially larger than those measured previously- 
a five-fold increase at the IS/OS and a threefold increase at COST, peaking $\sim 2$ min after bleach. They also showed that the rod OS elongated by approximately $2 \mu \mathrm{m}$, or about $10 \%$ of the OS length, peaking 3 min after bleach. Additionally, they showed that both of these responses were reduced in a transducin knockout mouse, providing strong evidence that the observed responses are associated with a process downstream of transducin activation.

Lu et al. used a custom SD-OCT system to measure similar light-evoked changes in humans, primarily in rods (96). They acquired volumetric scans over a $6 \mathrm{~mm}$ square region of the retina, centered about the fovea. After bleaching between $23 \%$ and $96 \%$ of rhodopsin (and similar amounts of cone opsin) using a Maxwellian view stimulator with a $528 \mathrm{~nm}$ source, they took measurements every 20 to 60 seconds for 30 minutes. They observed an immediate elongation of the cone OS of $\sim 50-400 \mathrm{~nm}$ that scaled with bleaching fraction, followed by a return to baseline within a few minutes. In rods they observed a very different dynamic, with elongation initiating immediately after the bleach, but peaking $\sim 7$ min after the bleach. The time course of the rod elongation was slower (by a factor of $\sim 2$ ) than what was found by Zhang et al. in the mouse. The axial resolution of the system they used was $3 \mu \mathrm{m}$, but by averaging over a large field of view (FOV), they were able to estimate OS length changes an order of magnitude smaller. While their system is a custom, research-grade OCT, it is very similar to clinical systems. Their results offer some hope that commercial OCT systems in the near future may have ORG capabilities.

\section{Phase-sensitive adaptive optics OCT (AO-OCT)}

The advent of AO OCT offered a new way to study the three-dimensional morphology of the retina at the cellular level $(16,17,19-21)$. Unlike other ophthalmic imaging modalities, in OCT the phase of the light backscattered from the retina affects the acquired signal. In TD-OCT, which records the envelope of the interference fringe, specialized algorithms and/or equipment are required to resolve the phase. In SD-OCT and swept-source (SS) OCT, however, the fringe is recorded and thus the phase is readily available as the arctangent of the complex, Fourier transformed fringe. Soon after the development of SDOCT, investigators realized that by tracking the phase of the OCT signal generated by an in vitro sample, motion with amplitude much smaller than the axial resolution could be detected and measured (97).
Although OCT permits measurement of the backscattered light's phase, the precision with which a single scatterer can be axially localized is limited by many factors: air currents, vibrations in the system, and-in the case of in vivo imaging, movements of the head and eye. The resulting shifts in the phase of the backscattered light can be much larger than the cellular dynamics of interest, and bury the latter in noise. Making matters worse, any movement larger than half the light's wavelength causes further ambiguities due to phase wrap.

Our group observed that while the absolute phase of light returning from a given surface was subject to such phase errors, phase differences between scatterers would not suffer from such artifacts (98). Drawing on estimates of OS elongation velocity from earlier AO-FI work (79), we determined that the AO-OCT system's speed of 1-2 Hz for a FOV of $1^{\circ}$ square (99) would likely be insufficient for measuring light-evoked OS elongation. Instead, to validate the approach, we measured the much slower process of OS disc renewal, and found that it showed rates of OS renewal similar to what we had previously measured using AO-FI (69). It would be several years before scanning OCT systems would become fast enough to measure lightevoked changes, either through multiplexing (100) or the development of swept sources with $\mathrm{MHz}+\mathrm{A}$-scan rates (101,102).

Investigators developing full-field (FF) SS-OCT systems demonstrated that if implemented using sufficiently fast two-dimensional complementary metal oxide semiconductor (CMOS) sensors, FF-SS-OCT systems could acquire A-scans at effective rates an order of magnitude faster than scanning systems $(103,104)$. Because the retina is imaged onto a 2D sensor, images don't suffer from the eye motion artifacts (warp) observed in scanning implementations. FFSS-OCT suffers from some inherent limitations compared to its raster scanning cousin, due to common path coherent artifacts and coherent spatial cross-talk between pixels in the sensor which reduce its sensitivity. Pioneers in the field recognized that its sensitivity could be improved by using an off-axis reference mirror to reject autocorrelation noise (105). Others have investigated how spatial phase modulation in the image and/or pupil plane can reduce crosstalk $(106,107)$.

Investigators also recognized that OCT measures the complex electromagnetic wave at the sample, and thus can be used to reconstruct computationally the wave in the pupil and thus estimate and correct aberrations (108). While this approach, termed digital aberration correction 
(DAC), has been demonstrated to improve resolution in scanning OCT systems $(109,110)$, it cannot recover photons lost due to degradation of the lateral PSF and associated losses in coupling back into the interferometer. In FF-SS-OCT, those photons are not lost, but rather misplaced on the 2D sensor by optical blur; estimation of the pupil function and its aberrations allows the signal to be rearranged computationally, as if they had been focused onto the sensor with perfect optics. DAC also permits effective extension of the depth of field through independent defocus correction over the axial extent of the retina. By the same principle, it could permit spatially varying correction of aberrations, effectively expanding the isoplanatic patch that fundamentally limits hardware AO. DAC is a computationally intensive algorithm, and has thus far been made tractable by limiting the order of the correction's basis set and/or the size of the computed pupil. Further, the ability to estimate and correct aberrations computationally is limited by the SNR of the OCT image. In hardware AO, this limit is imposed by the SNR of the wavefront measurement, and it is not clear which has the greater sensitivity.

Researchers working in FF-SS-OCT showed that their systems were fast and sensitive enough to measure lightevoked changes in OS length, and that DAC would allow them to resolve these changes in single cells. Leveraging these capabilities, they measured, for the first time, the elongation of cones in response to visible stimuli (111), and showed that cones continue to elongate for a few hundred milliseconds following the flash, before returning to their unstimulated length within a few seconds. Their data suggested that the elongation velocity was independent of stimulus dose-a result that differs from subsequent experiments (see below). They also observed an initial contractile phase, in which the OS contracted by $5-10 \mathrm{~nm}$ within the first $20 \mathrm{~ms}$, a result that would be reproduced by Zhang et al., 2019 and Pandiyan et al., 2020 (below). The system was used to measure light-evoked OS elongation in peripheral cones, but not in the smaller foveal or parafoveal cones. The distinction is relevant for DAC, since the computational tractability of digital aberration correction is greatly impacted by the desired resolution.

The same investigators were later able to show that light-evoked swelling occurs in the ganglion cell layer, slightly displaced from the stimulated region by the splayed neural circuitry, as well as a possible response from the rod OS layer (112). Most ORG research has been focused on the $\mathrm{Ph}$, because the high SNR of the Ph bands permits very high phase sensitivity. Investigators studying $\mathrm{Ph}$ responses are presumably interested in other retinal neurons as well; specific ORG methods, once honed on the $\mathrm{Ph}$, are likely to be employed to measure responses of other retinal neurons as well.

Soon after, our group developed the fastest AO-OCT system at the time, capable of acquiring A-scans at a rate of 1.6 $\mathrm{MHz}$ and video rate $3 \mathrm{D}$ imaging over a $1^{\circ}$ square FOV. Leveraging a closed-loop hardware AO system, it was used to measure light-evoked changes in $\mathrm{Ph}$ within a few degrees of the foveal center (113). The unprecedented speed was owed to a Fourier-domain mode-locked laser (101) operating at $1,060 \mathrm{~nm}$, a wavelength which limits the lateral resolution to about $3 \mu \mathrm{m}$ through a $6.75 \mathrm{~mm}$ dilated pupil. As such, cones at the center of the fovea (foveola) and rods were not well resolved. Nevertheless, cones at the edge of the fovea $\left(2.5^{\circ}\right)$ were imaged, and light-evoked elongation was reliably measured. Contrary to Hillmann et al., the data shown in Azimipour et al. suggests a dosedependent variation in the elongation velocity. Moreover, we made a novel observation of axial rearrangement of scattering layers, with an aspect of the IS/OS band moving distally after stimulus, while an aspect of the RPE moved proximally. Soon after, the measurements of light-evoked changes in OS length were reproduced by Zhang et al. (114), who used a similar approach to measure differential responses among cones to colored stimuli, in order to classify them by spectral class. Like Hillmann et al., Zhang et al. observed an initial contractile phase lasting about $10 \mathrm{~ms}$. They observed that the velocity and maximum excursion of the cone OS tip scaled with photopigment bleaching, providing important evidence that the observed responses were correlates of $\mathrm{Ph}$ function.

These demonstrations of the ability to probe cone function in the central retina were important, since impairment of these $\mathrm{Ph}$ [e.g., in age-related macular degeneration (AMD)] tends to have a more significant impact on quality of life than peripheral cones or rods. However, rod dysfunction is thought to precede cone dysfunction in a number of important diseases (115), as demonstrated by studies using dark adaptometry in early AMD $(116,117)$. Rods are smaller than cones and thus more difficult to resolve; the complete rod mosaic had never been resolved using AO until after nearly fifteen years of development and optimization of optical systems (118). Like cones in the center of the fovea, rods are too small to be resolved using the fast-scanning system developed by Azimipour et al., due to its $1 \mu \mathrm{m}$ imaging wavelength and 
subsequent lateral resolution of $3 \mu \mathrm{m}$ through a $6.75 \mathrm{~mm}$ dilated pupil. Images we acquired with that system revealed clusters of what looked like rods, but whose identity could not be confirmed. To circumvent this limitation, we added an $840 \mathrm{~nm}$ SLO channel to the system to acquire coextensive AO-OCT volumes and AO-SLO images, the latter having about $20 \%$ better resolution (119). Using the combined system, we were able to show light-evoked elongation of rods and cones together and compare the dynamics of the two classes of cell (120). We also reported axial rearrangements of structure near IS/OS and RPE in rods, similar to those we previously reported in cones. We showed that cones elongated more rapidly than rods, but elongation was observed in rods at much lower fractions of photopigment bleaching (e.g., 0.007\% bleach). Because the mechanism of OS elongation is not yet known, direct comparisons between these results and psychophysical or electroretinographic literature are premature. Nevertheless, it is noteworthy that these results are congruent with our estimates of the respective temporal bandwidth and sensitivity of cones and rods. Based on the sigmoidal model we proposed to characterize the relationship between percent of rhodopsin bleached and rod OS elongation, the response saturates (99-99.5\%) at rhodopsin bleaching levels between $5-10 \%$. At saturation, the rods appeared to elongate by approximately $150 \mathrm{~nm}$, significantly less than the elongation reported in mice by Zhang et al. (94) and humans by Lu et al. (96). The latter studies used the amplitude of the OCT image to assess length, without the benefit of cellular resolution. While it is not yet known why these studies do not conform with one another, it is possible that measurements of length using either method could be impacted by the band movements reported by Azimipour et al., or that the axial and lateral aggregation of the conventional OCT signal affects its measurements in ways that phase-sensitive AO-OCT avoids.

As interest grows in this emerging functional assay, several efforts are underway to improve it and make it suitable for clinical translation. In FF-SS-OCT with DAC, one of the major hurdles is the computational tractability of optimizing the digital pupil function. This has, in general, been approached in an iterative way, but the high dimensionality of the aberration space is likely to make the approach impractical in clinical applications, where real-time feedback on image quality is paramount. Some investigators have tried to improve DAC by measuring wavefront error using local estimates of tip and tilt in the digital pupil, analogous to how Shack-Hartmann wavefront sensors estimate local tip and tilt in the physical pupil (121-123). More recently, some of those investigators have shown that aberrations can be estimated more rapidly and with higher spatial bandwidth through digital shearing of the computed pupil function (124). Preliminary results have been encouraging, and suggest that DAC could in principle be performed quickly enough for a clinician to adjust imaging parameters such as focus, pupil centration, and axial alignment, or for the imaging system to optimize these automatically. The very high-volume rates (and effective A-scan rates), phase stability, and freedom from fixational movement artifacts make FF-SS-OCT an attractive option, because they all enable tracking of the fastest light-evoked deformations. The additional promise of DAC to produce sharp images over extended regions in three dimensions makes it especially attractive compared to traditional scanning AO-OCT systems with their large numerical apertures, limited depths of field, and isoplanatism. However, the sensitivity of FF-SS-OCT systems has not yet approached that of raster scanning systems, which may be due to the significantly lower number of photons used to generate each A-scan and the coherent cross-talk noise.

The sweep rate of FF-SS-OCT systems is orders of magnitude lower than that of scanning SS-OCT systems, which amplifies the effects of disturbances to the interferometer due to system vibrations, air currents, or eye movements, and this may place an additional limitation on FF-SS-OCT sensitivity. Pandiyan et al. developed a novel line-scanning AO-OCT system to try to combine benefits of both SD-OCT and FF-SS-OCT (125). In their approach, the retina is illuminated with a line of broadband light, which is imaged onto a 2D sensor through a diffraction grating, such that one dimension on the sensor corresponds to spatial extent (along the line) and the other to wavelength, as the light is dispersed by the grating. Each 2D image is then transformed into an OCT B-scan, as would a series of spectral measurements along the fast scan in a traditional system. Their approach yields B-scan rates higher than traditional scanning systems-up to $16 \mathrm{kHz}$, corresponding to $8 \mathrm{MHz} \mathrm{A}$-scan rates-but lower than the effective A-scan rates of FF-SS-OCT systems $(111,126)$. With this system, they were able to measure light-evoked deformations of the cone OS, and use these to spectrally classify cones. Like Hillmann et al. and Zhang et al. before them, they identified an initial contractile phase of the response. They showed that both the time-to-peak and the contraction amplitude scaled logarithmically with fraction of photopigment bleaching, even above levels that 
saturate the phototransductive process, indicating that this process is associated with isomerization of photopigment $(127,128)$. They hypothesized that the contractile phase is due to the early receptor potential (ERP) visible in electroretinographic recordings, especially with bright stimuli $(129,130)$.

To overcome the computational demands of highorder DAC, our group developed an FF-SS-OCT system with hardware AO (126). The system was demonstrated to acquire $\sim 1^{\circ}$ retinal volumes at $200 \mathrm{~Hz}$, sufficient for measuring both the initial contractile phase and elongation of cones, with the potential lateral resolution to measure rod changes as well. Over smaller fields of view, the system was shown to collect $3 \mathrm{D}$ images of the $\mathrm{Ph}$ at rates up to $1 \mathrm{kHz}$, demonstrating the potential to characterize OS deformations with exquisite temporal detail. With the reference arm blocked, the system is a kilohertz AOFI system with fine control over effective coherence length, and thus could be useful in resolving some of the ambiguities observed in functional AO-FI and AO-SLO experiments described above.

\section{A taxonomy of functional signals}

The functional signals described above have translational potential, and their known or hypothetical origins can be enumerated as follows:

\section{Signal 1: backscattering from OS discs}

Increase in backscattering from the OS discs, due to redistribution of charge and/or mass about the disc membrane and subsequent changes in the interdisc spacing; the biochemical processes most implicated are the binding of $\mathrm{R}^{*}$ to transducin, the concomitant movement of the transducing $\alpha$ subunit away from the membrane, and interactions between transducin and PDE.

\section{Signal 2: backscattering from OS boundaries}

Increase in backscattering from the IS/OS and outer segment tips (OST), hypothetically due to a change in the refractive index of the OS or ultrastructural rearrangements at those boundaries.

\section{Signal 3: band rearrangement}

Axial rearrangement of scatterers, visible in images from
Bizheva et al., 2006 (85) and first described by Azimipour et al. $(113,120)$; the outward movement of an aspect of the IS/OS was attributed to an abrupt change in disc spacing or refractive index, which moves distally over time; the inward movement of an aspect of the RPE was hypothetically attributed to passive movement of the RPE's melanosomes toward its apical boundary, possibly within the microvilli.

\section{Signal 4: OS deformation}

Fast (5-10 ms) contraction of the OS, hypothetically due to changes in membrane tension in the ERP; Slower $(0.1-10 \mathrm{~s})$ elongation of the OS, hypothetically due to osmotic rectification of shifting concentration gradients, possibly transducin subunits released from disc membrane (94).

The capacity to image and quantify functional processes in the retina would have significant impacts on our understanding of retinal disease and its clinical management. The ORG now seems poised to play an important role in our understanding and management of diseases affecting $\mathrm{Ph}$ function. Without empirical comparisons between clinical functional standards (visual acuity, contrast sensitivity, perimetry, and ERG) and the ORG, it's impossible to say with certainty which is more sensitive to disease-related dysfunction. Thus, there is a need for experiments comparing these methods.

\section{Light-evoked changes in amplitude of light scattered by the retina}

Given its association with the activity of transducin, disc backscattering ("Signal 1" above) could be a way to identify congenital or acquired defects in that part of the phototransduction pathway. GNAT1 mutations, for instance, can cause some forms of congenital stationary night blindness by interfering with rod transducin (131-133). Similarly, forms of cone dystrophy are associated with mutations in GNAT2, which encodes the $\alpha$ subunit of cone transducin (134). Because transducin-PDE interactions are downstream of photopigment isomerization, diseases impacting the RPE (e.g., AMD) or visual cycle (e.g., Stargardt's disease) may manifest as reductions in the scattering signal. Because dissociation of transducin- $\alpha$ is metabolically demanding, cells with limited supplies of nutrients or oxygen, e.g., due to reduced permeability of Bruch's membrane, may display a reduction in light-evoked scattering. Although the binding and dissociation signals have opposite signs, as shown by Vuong et al., 1984, when 
the illumination is axial—as it is in typical human retinal imaging - the binding $(\mathrm{P})$ signal dominates, resulting in an overall increase in scattering, an idea echoed by Arshavsky et al., 2002.

However, as Hill observed decades earlier, the relationship between light scattering and the underlying disc changes is likely to be obscure (32). Even if lightevoked OS scattering becomes a clinically meaningful biomarker, much work remains to be done to understand the biochemical mechanisms that underlie it.

Changes in the intensity of the IS/OS and OS tips ("Signal 2" above) have been shown in animal models and humans, with or without accompanying changes in the OS length. Axial localization of these changes has been permitted thus far only by OCT, although they may have affected the numerous early studies of scattering in suspensions of rod OSs or axial measurements of explants. One trouble with these findings is that measured changes are inconsistent among studies. These inconsistencies may be due to interspecific or methodological differences, and may be resolved by further experiments. OCT is a rapidly evolving technology, and so too are the anatomical attributions of features seen in OCT-the most recent measurements (e.g., Zhang et al., 2017) have the greatest benefit of hindsight. With the exception of the two earliest measurements (Yao et al., 2005 and Bizheva et al., 2006), a common finding has been that backscattering from the IS/OS band increases after light exposure $(89,90,92)$. The simplest interpretation of this finding is that refractive index mismatch at IS/OS becomes greater, consistent with water movement out of the OS and attendant increase in the OS refractive index and IS/OS index mismatch. This also conforms with earlier hypotheses that light stimuli cause a contraction of the interdisc spacing, which would cause an increase in lipid density and refractive index. However, it is inconsistent with other studies which suggest that light stimuli cause swelling of the OS.

\section{Light-evoked rearrangements of subcellular structures}

The rearrangement of bands ("Signal 3", above) is the only one of these phenomena described by my group alone $(113,120)$, and should thus be regarded skeptically until it is reproduced. Nevertheless, it is an interesting one because it could potentially reconcile some inconsistencies among other findings. If the movement of part of either band (outward from IS/OS or inward from RPE) affects the amplitude of the residual, non-moving component, then the measured increase and decreases in scattering could be due merely to differences in segmentation methods, thus reconciling the early OCT findings of Yao et al., 2005 (84) and Bizheva et al., 2006 (85) with more recent OCT measurements of scattering changes from the outer retinal bands. A tightly segmented IS/OS band might display a reduction in scattering while a more liberally segmented band might display an increase. The same could be said of the inward movement of a part of the RPE band. Because the latter is very close to both the ROST and the COST, its rearrangement could create significant artifacts in measurements of scattering changes from those layers, not to mention from RPE itself.

While the movement of an extra IS/OS band was a novel observation, the existence of "extra reflectors" within the OS had been noted before in AO-OCT images, and these have been observed to move slowly as a function of disc renewal (135). If we assume that the moving, visible bands are either a distinctive OS region or a boundary between two OS regions, where the regions are defined by differences in disc packing or distribution of mass about the disc membrane, the observed diversity may also underlie the finding by Chabre et al., 1973 that bright stimuli broaden the $\mathrm{X}$-ray diffraction peaks, indicative of an increase in disorder in the lattice (48). Chabre et al. claimed that the effect was not consequential owing to the unnatural level of bleaching required to observe it. Azimipour et al. showed that the effect was most pronounced after bleaching $70 \%$ of photopigment, but also showed that it was visible after much dimmer flashes (113).

\section{Light-evoked changes in OS length}

The last category of observation, changes in OS length ("Signal 4", above), includes both contraction and elongation of the OS, in succession. Zhang et al., 2017 provide a hypothetical explanation for light-evoked elongation, attributing it to the dissociation of the G- $\alpha$ and G- $\beta \gamma$ subunits of transducin from the membrane-a net increase in solute, amplified by the fact that a single $\mathrm{R}^{*}$ can activate 16 transducins in the mouse (136)—followed by osmotic rectification of the resulting gradient. If true, lightevoked elongation could be an effective indicator of diseaserelated changes in photopigment availability (e.g., due to ABCA4 mutations or other visual cycle impairments) as well as metabolic or energetic dysfunction that interferes with $\mathrm{R}^{*}$-transducin dissociation, and also benefit from the 
amplification in transducin interaction.

The hypothetical attribution of OS swelling to transducin activity conforms with recent measurements of rod elongation as well. Rod elongation has been shown to saturate in terms of maximum length $(94,120)$ and maximum velocity (120) at rhodopsin bleaching levels between $10-15 \%$, consistent with reported ratios of rhodopsin to transducin, and also consistent with earlier observations of functional saturation in animal rods. This is a stoichiometric simplification, ignoring the reaction rates and other biochemical kinetics, but it's within a factor of 2 of more sophisticated estimates (136).

Several observations from early experiments suggest that with respect to clinical translation, experiments conducted in vivo or on intact retinal explants may be more valuable than those conducted on isolated rods or $\mathrm{Ph}$ separated from RPE. Chabre and Cavaggioni noted that the observed increase in interdisc spacing occurred only in the intact retina; in isolated rod OSs, no such signal was present $(48,49)$. Uhl et al. showed something similar regarding the superposed positive $(\mathrm{P})$ and negative $(\mathrm{N})$ signals, that the $\mathrm{N}$ signals are only present when the plasma membrane of the rods are broken and that in the intact rod only the $\mathrm{P}$ signal is present (52). No reason was given, but it is plausible that the GTP concentrations in suspensions of discs was insufficient to permit the GTP-dependent dissociation of transducin- $\alpha$ and transducin- $\beta \gamma$, and thus the $\mathrm{N}$ signal. Vuong et al. showed that the rapid $\mathrm{P}$ signal is isolated when illumination is axial (60), consistent with the simplified view of scattering advocated by Arshavsky et al. (65).

If the initial contractile phase proves to be an optical signature of the ERP (128), then it may be sensitive to important pathologies of the $\mathrm{Ph}$ (137). Reduced amplitude and faster completion of the contractile phase could be indications for retinitis pigmentosa, and reductions in amplitude could be an indication of a number of inherited $\mathrm{Ph}$ dystrophies, central serous retinopathy, Stargardt's disease, or AMD (137). Because the ERP is thought to be a side-effect of opsin activation, it is possible that the contractile phase could be used to detect deficits in the visual cycle as well. On the other hand, because it is upstream of the amplification steps in phototransduction, and because its smaller amplitude necessarily reduces its SNR, subtle disease-related changes in the contractile pattern may be difficult to detect.

It is interesting to note the conformity of much of the work done in this area, especially as it relates to the activity of transducin. However, these connections are speculative, and highlight the importance of doing further experiments with modern imaging tools. In humans, studies of well-characterized retinal diseases could serve as natural experiments, showing how ORG measurements are affected by known modifications in the underlying biochemical and biophysical processes. In parallel, studies of animal knockout models could confirm emerging hypotheses.

\section{Realizing the ORG's clinical potential}

The translational and clinical potential of the ORG is significant, in particular in the areas of disease diagnosis and staging and endpoints for clinical trials. The ORG may improve the sensitivity and specificity of disease diagnosis and progression. Given its potential to improve vision care, translation of one or more of these technologies to the clinic is an urgent need. Each implementation of the ORG thus far has advantages and disadvantages, and these should be weighed carefully as the research and commercial communities decide which to invest in.

ORG studies thus far have been limited to volunteers without severe retinal disease. As all investigators in the field know, imaging patients with vision loss and retinal disease poses unique challenges: they may not be able to fixate well; retinal lesions or edema may significantly degrade image quality; head stabilization with dental impression bite bars and forehead rests may not be tolerated by patients; long periods of dark adaptation may make studies difficult to schedule.

When ORG methods are proposed for clinical work, the needs of clinicians must be considered as well. While the pioneering work on phase-sensitive, single-cell ORG recordings is impressive, at present the technique cannot provide images without hours of signal processing. On the other hand, potentially faster methods-such as coherent flood imaging-may have substantially poorer sensitivity to disease- or therapy-related changes in function.

For the sake of patients and clinicians, the trade-offs presented by each of the methods should be weighed carefully.

\section{Potential avenues for clinical translation}

The capacity to measure the light-evoked phenomena described above vary among current clinical and research modalities. The major clinical and research modalities currently available are described below, along with assessments of their translational value. 


\section{Fundus photography}

Abràmoff et al., 2006 showed that a clinical fundus camera is capable of revealing light-evoked reductions in fundus reflectance. The amplitude of these changes was $0.14 \%$, with comparatively high standard deviation. Although their result was significant, the high variance suggests that the sensitivity to disease-related losses may be small. On the other hand, the required equipment is minimal—a clinical fundus camera modified to incorporate a NIR illumination source and green stimulus source.

\section{Coherent ophthalmoscopy (FI or SLO)}

Closely related to laser speckle contrast imaging, coherent FI and SLO offer the capacity to amplify microscopic deformations of tissue, such as those caused by compression or expansion of discs, or contraction and elongation of the OS. This capacity has been explored with AO (see below), but in conventional retinal imaging its applications have largely been limited to the identification of blood flow and tissue perfusion. In potential ORG applications, retinal blood flow would be a significant source of confounding signal, and strategies for differentiating light-evoked deformations from flow would need to be developed. Moreover, because FI permits no confocal gating, deformations from all depths in the retina would contribute to the signal. With sufficiently fast sampling and careful temporal analysis (e.g., as is done in full-field ERG), however, we may be able to differentiate contributions from different cells. Due to the comparatively low cost, small data size, and minimal processing requirements of such a system (compared with the AO and OCT systems described below), further investigation of this approach is warranted.

\section{Clinical and conventional OCT}

Conventional (non-AO) research-grade OCT systems continue to improve, and these improvements are bound to make their way into commercial/clinical OCT and OCT angiography (OCTA) systems. As these systems become faster and more sensitive, their ability to measure ORG responses will improve. Because of the wide deployment of these systems, and because OCT is now a standard of ophthalmic care (138) and OCTA is rapidly becoming one, an ORG method based on conventional OCT could have a great and immediate impact on translational research. This is especially true because in principle it has access to all four of the functional signals described above. It has been used to reveal scattering from the OS or its terminal reflections $(84,85,88-90,92,94)$ and also the length of cells or thickness of layers $(93,94)$.

A question remains as to whether OS deformations ("Signal 4", above, and Figure 3) can be detected in the phase of the conventional OCT signal, where because of the large imaging spot size, any single A-scan contains contributions from the reflective bands of multiple cells. Instead of resolving individual $\mathrm{Ph}$, the $\mathrm{Ph}$ mosaic manifests as a speckle field (139). In principle, the phase of both reflective surfaces is deterministic, even when blurred by a relatively low-resolution instrument, and stimulusevoked changes in the optical path length should generate reproducible patterns of relative phase change. Further still, multiple A- or B-scans could be combined on the same basis. This was recently demonstrated over an area ranging between $0.07^{\circ} \mathrm{sq}$ and $0.27^{\circ} \mathrm{sq}$ retinal region at $7^{\circ}$ eccentricity, without AO (125). At such large eccentricities, where rods significantly outnumber cones, rod and cone responses could potentially be differentiated by axial segmentation (120), even if the cells are not resolved.

Because phase-sensitive OCT has the potential to reveal OPL changes on the order of $\lambda$ (wavelength)/100, it is highly susceptible to sources of phase noise such as eye movements and system vibrations. However, owing to the rapid development of OCTA methods in the past decade, especially phase variance (140) and complex correlation (141), algorithms for assessing and correcting phase noise have been extensively developed. Additional numerical approaches for reducing phase noise have been proposed in the FF-SS-OCT community (142) and computational OCT (143).

\section{AO ophthalmoscopy (FI or SLO)}

Most of the AO ophthalmoscopy methods described above have utilized partially coherent superluminescent diodes (SLDs) or lasers, and should thus be considered coherent imaging, subject to signal modulation due to shifting of common path lengths. Although some disagreement remains among measurements using coherent FI and SLO, and thus among hypothetical explanations for the observed effects, there is no doubt that light stimuli elicit changes in the apparent reflectance of photorecpetors under coherent, NIR illumination. At present it cannot be ruled out that these changes are due to interference between reflective surfaces within the OS. The work by Rha et al. (80), 


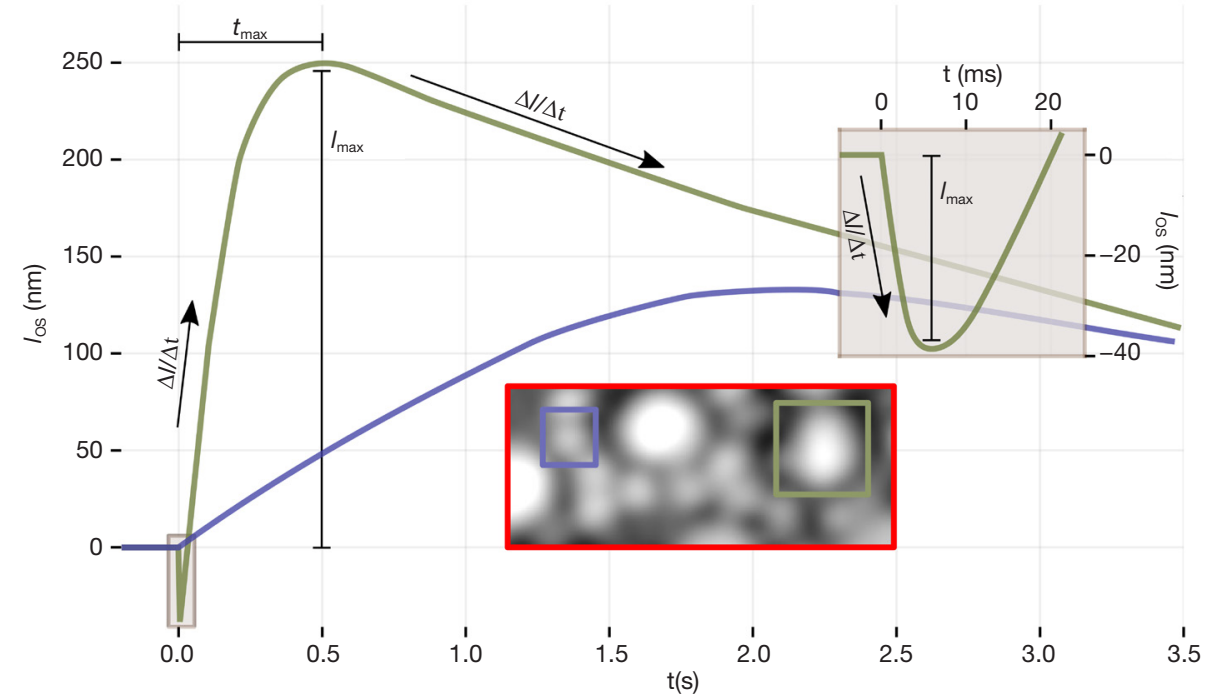

Figure 3 Photoreceptor deformations evoked by light stimuli, measured with phase-sensitive OCT with AO or DAC. As has been shown with both full-field and scanning OCT systems with AO or digital aberration correction, the length the photoreceptor outer segment changes in response to light stimuli. These systems have spatial resolution sufficient to individuate cone (and sometimes rod) Ph. In the red inset is shown a small portion of an en face projection through the rods and cones, collected using our AO-OCT-SLO system. The green and blue boxes highlight single cone and rod Ph. The plot depicts the concepts and key features of the cone (green) and rod (blue) responses to flashes bleaching $12 \%$ and $0.8 \%$ of photopigment, respectively. The cone response $\left(l_{\mathrm{OS}}\right.$, green) exhibits an initial contractile phase (inset) followed by rapid elongation and slower return to baseline. The amplitude of the rod response (blue) is greater relative to bleaching, but rises and falls more slowly than in cones. An initial contractile phase has not yet been observed in rods. Several parameters are labeled on the cone curve: maximum contraction and elongation $\left(l_{\min }\right.$ and $l_{\max }$, respectively), as well as slopes $\Delta \mathrm{l} / \Delta \mathrm{t}$ for the contraction, elongation, and recovery portions of the curve. All of these parameters appear to be dependent on the number of opsin photoisomerizations, in both rods and cones. The dose dependence of time-to-rise $t_{\max }$ has yet to be established; in some experiments it appears to be dose independent. Each parameter may have unique sensitivity and dynamic range, both of which are critical for assessing translational impact. These biomarkers should be studied carefully in healthy subjects before designing disease studies or endpoints. Conceptual plots based on unpublished data from separate experiments in my lab using (I) full-field AO-OCT to measure L-/M-cone responses to a flash bleaching $12 \%$ of each cell's photopigment; (II) confocal, scanning AO-OCT to measure rod responses to a flash bleaching $0.8 \%$ of rhodopsin. OCT, optical coherence tomography; AO, adaptive optics; DAC, digital aberration correction; $\mathrm{Ph}$, photoreceptors; SLO, scanning light ophthalmoscopy.

Bedggood and Metha (81), and Cooper et al. (82) together suggest that the coherence length of the source need not be longer than the cone OS to observe light-evoked changes in OPL. This apparent contradiction may be resolved by the recent observation that there are multiple sources of backscattering within the cone OS, and that the positions and backscattering of these surfaces change after light stimuli $(113,120)$. The IS/OS, COST, and other surfaces within the OS or in the RPE may all contribute to the interference signal. This hypothetical dependence of the observed effects on illumination coherence and cellular activity is described in (Figure 2).

Some have hypothesized (89) that the oscillations arise not purely from relative motion among reflectors in the OS, but rather from the appearance of an additional scattering region, which is consistent with the numerous observations of light-evoked scattering changes in suspensions of rod OSs or rod OS discs, some of which are described above. This hypothesis assumes that the new scatterers in the OS produce a sum of waves with stable phase. This would be a tall order for scatterers free to diffuse in three dimensions, e.g., those in suspension, but could be a realistic assumption for scatterers whose motion is constrained by the structure of the discs.

It is tempting to reconsider observations of short coherence oscillations in light of the observation of band rearrangements ("Signal 3", above). The secondary IS/OS band was observed to migrate away from the IS/OS after 
light stimulus, and its excursion was (qualitatively) observed to be a function of stimulus dose $(113,120)$. When cone bleaching was $<10 \%$, the new band appeared to migrate less than $10 \%$ of the OS length, and when the bleaching was much higher $(70 \%)$, it was observed to move about half the OS length. It was not as bright as the original IS/ OS nor the COST, but its amplitude was $20-50 \%$ of those, which suggests an interference fringe contrast only $20-30 \%$ smaller than that arising from interference between IS/ OS and COST. In short, the moving aspects of the IS/OS and RPE could make significant contributions to common path interference signals, even when the coherence length is short. Azimipour et al. observed these moving extra reflections significant fractions of cones and rods $(113,120)$, which is consistent with the short coherence findings.

A challenge faced by coherent en face methods is that quantification of the responses is a difficult inverse problem. The interferometric nature of the signal means that the initial direction of change depends on the random initial phase of the fringe-or baseline OS length. Jonnal et al., 2007 were able to observe light-evoked oscillations (79), but because the oscillation frequency varied significantly over time, standard frequency analysis tools were not of quantitative value. We were able to quantify changes only grossly, by showing that the temporal standard deviation of the cone amplitude was higher in stimulated retina than unstimulated retina (79). Rha et al., 2009 (80) addressed this issue by classifying and counting cones by the shape of their light-evoked change (namely, oscillating, rising, falling, or flat). Cooper et al., 2017 (82) approached the problem by computing the variance among cells over time, which offered a reproducible metric at the expense of cellular resolution (82). To address variations in polarity and slope of change in their animal work, Yao et al. have generally employed a method that quantifies response by counting the number of "active" pixels, i.e., those which change in intensity, either upward or downward $(67,144)$.

One weakness of such approaches is that the quantitative relationship between the measured value and the underlying physical change is obscure. Additionally, in all of these approaches, the light-evoked phase shifts manifest as a reflectance oscillation whose number of cycles corresponds to the total path length change. However, for sinusoidal oscillations-even chirped ones-the maximum variance is achieved after one cycle, which implies that the dynamic range of such measurements is limited to elongations less than one cycle [corresponding to $\lambda /(2 \mathrm{n})$ ]. Coarse metrics such as the standard deviation of reflectance over time (79) or among cones (82) would necessarily have much smaller dynamic ranges, since they're correlated with elongation only over a fraction of one wavelength, while light-evoked elongation can span multiple wavelengths. By comparison, sufficiently fast AO-OCT has unlimited dynamic range for measurement of elongation.

One possible way to quantify common path interference signals without sacrificing dynamic range or resolution would be to piggy-back on the results of phase-sensitive AO-OCT. The latter technique has been demonstrated and reproduced in cones in a number of labs over the past few years, with remarkable consistency of observations among subjects and imaging systems. The two reports of rod ORG responses thus far $(112,120)$ are inconsistent with one another, but further experiments are likely to reveal a predictable response. An over-damped harmonic oscillator model proposed by Azimipour et al. has been shown to fit cone responses well (145), and could potentially be used to identify unique solutions to the oscillation pattern. The capacity to measure both en face changes and elongation simultaneously (120) could prove invaluable in such an effort.

On the other hand, noise arising from the tails of the coherence function and/or unstable secondary reflections in the OS could greatly reduce the sensitivity of this method to disease-related defects (Figure 2). Noise in the illumination source's spectrum makes the coherence function difficult to estimate, and would impede efforts to uncover the underlying patterns of contraction and elongation. Complicating matters further is the fact that secondary reflectors in the OS are not visible in en face images, and thus their role in the resulting oscillations would not be known.

\section{Phase-sensitive AO-OCT}

Based on spectral domain phase microscopy (97) and OCT relative-phase imaging (98), this is the latest entrant to the field of ORG, with fewer than ten papers published since $2016(111-114,120,125,127)$.

Because AO-OCT provides axial and lateral localization of backscattered light, with cellular resolution, it combines the advantages of several competing approaches because it permits measurement of stimulus-evoked changes in both the amplitude and phase of backscattered light. Thus, in principle it has the ability to measure stimulus-evoked changes in light scattered by the OS, IS/OS, COST, and ROST (Signals 1 and 2, above), without ambiguity about 
their origin. Because it can resolve scattering objects within cells, it is capable of detecting the motion of subcellular structures, such as the outward movement of a secondary IS/OS band, and inward movement of an RPE band (Signal 3, above). Most importantly, it has the ability to measure light-evoked deformations of the OS (Signal 4, above), with a theoretical precision of a few nanometers. The patterns of deformation found in cones and rods are summarized in (Figure 3), along with potential methods of signal quantification. While methods of quantifying and modeling the resulting deformations are still being developed, the dynamic range of $\mathrm{Ph}$ responses is several orders of magnitude (120), which suggests a high sensitivity to disease-related changes. Conventional OCT offers many of the same advantages, but it has not yet been used to measure deformations as small as those seen using phasesensitive AO-OCT.

In spite of the relative paucity of reports using this technique, the results of different groups have conformed well. This is in part because the observed phase changes require few assumptions to interpret correctly, and different from approaches based on en face imaging, where the detected signal consists of a mixture of the four signals listed above. Much a priori knowledge about both the imaging and biological systems must be dispatched in order to separate these components, making errors in interpretation more likely.

Investigators using phase-sensitive AO-OCT have reported the ability to see phase changes in single cells. As a technical achievement, this is of interest, but it is worth noting that all of the investigators have pooled responses from multiple cones (or the same cone over multiple measurements) to make robust, reproducible measurements. This highlights the multiple trade-offs between experiment duration, spatial resolution, and sensitivity that cannot at present be avoided. Especially in the context of clinical translation, it is critical that these trade-offs be evaluated.

While the advantages of AO-OCT as an ORG approach are numerous, unlike fundus photography and conventional OCT, it requires specialized equipment-either an AO system or a camera sufficiently fast for FF-SS-OCT, and therefore its cost of construction and operation is substantially higher than the former. Moreover, the data volumes produced by these approaches are very large. The AO-OCT and FF-SS-OCT systems constructed in my lab produce $3.6 \mathrm{~Gb} / \mathrm{s}$ and $18 \mathrm{~Gb} / \mathrm{s}$, respectively, and a single ORG experiment can require days of data processing and terabytes of storage space. While DAC is an attractive alternative to AO, especially as the cost of high-speed CMOS cameras continues to fall, it requires considerable additional processing time, which at present prohibits realtime visualization of the OCT image. DAC has also not yet been shown to be of use in recording ORG signals from foveal cones or rods. Nevertheless, as the cost of AO systems and high-speed CMOS cameras continues to fall, and computer hardware for high-speed analysis of big data sets becomes more available, these kinds of practical hurdles become easier to surmount.

While it is convenient to group scanning and full-field approaches together, as their capabilities largely overlap, it's worth noting some important differences. Scanning systems (both point-and line-scanning) benefit from confocality, and the rejection of multiply scattered light. They also do not suffer from cross-talk between A-scans due to spatial coherence. For this reason, and others, they have enjoyed significantly superior sensitivity thus far. While this sensitivity has not proven necessary for ORG measurements in $\mathrm{Ph}$, it may be required for studying less reflective neurons such as bipolar and ganglion cells. Additionally, full-field systems cannot be used in the line scanning mode reported by Zhang et al. (114) and Pandiyan et al. (127), and thus may not be capable of the very highest imaging rates. Fullfield systems, on the other hand, are less susceptible to eye movement artifacts, and are thus capable of measuring both the fast dynamics of the contractile phase and the relatively slower dynamics of the elongation phase in a single video of a few seconds duration.

While the physiological causes for contraction and elongation of the OS are not at present well understood, there is little ambiguity about the direction, magnitude, and time courses of the responses. That they have been observed in a mouse model, and have been disrupted by knocking out transducin (94) demonstrates the feasibility of correlative animal work. They have been shown to scale with stimulus dose, and to saturate, both in humans and animals-necessary characteristics for meaningful functional biomarkers. In a recent paper, Lassoued et al. were able to detect statistically significant reductions in cone elongation in patients suffering from retinitis pigmentosa (146), providing evidence that the elongation is sensitive to cone dysfunction.

Measurement of stimulus-evoked OS deformation using phase-sensitive AO-OCT appears to be a reproducible method with dynamic range and sensitivity likely higher than the other methods described above. Although it is more technically demanding and costly than other methods, 
its potential benefits seem to justify those costs, especially if they fall in the future.

\section{Conclusions}

The field of ORG is new, and the potential for significant discovery exists along all of the current avenues of investigation. However, the method that has produced the least ambiguous and best reproduced results has been phase-sensitive OCT with AO or digital aberration correction. The three-dimensional cellular resolution afforded by AO-OCT has permitted us to localize responses to single cells, and to identify the sub-cellular dynamics involved in the light-evoked response. This technology offers access to individual neural responses that had previously been available only to intracellular and singleunit electrophysiological methods. It has the potential to reveal a great deal about the mechanisms underlying ORG responses as well as ways in which disease may alter them.

While AO-OCT may be the best way to study the fundamental properties of the ORG, the current complexity, cost, size, and limited fields of view of these imaging systems cast some doubt on their widespread clinical deployment. Nevertheless, given the ORG's potential to accelerate the development of sight-saving therapies, there is an urgent need for robust and economical systems to be used in multi-site clinical trials and other clinical settings. For this purpose, several of the proposed technologies may be suitable. A natural choice is conventional OCT. It offers many of the advantages of AO-OCT, namely the ability to localize responses axially, and to measure stimulusevoked changes in the relative positions of the reflective retinal layers, albeit without cellular resolution. Similar to how angiographic capabilities have been incorporated into current OCT systems, optoretinographic capabilities could be added to next generation OCT systems, potentially requiring only the addition of a stimulus channel and requisite software. At this early stage, however, en face approaches—especially coherent FI and SLO-should be investigated further as well, as they offer the advantage of coherent amplification of stimulus-evoked deformations, along with smaller demands on data storage and computational bandwidth. Whichever methods are selected for clinical translation in the near term, it is important that the underlying mechanisms of the ORG continue to be studied using AO-OCT. Responses measured at the cellular level will lead to the development of better analytical approaches for low resolution signals and permit more informed interpretation of aggregate responses.

\section{Acknowledgments}

I would like to acknowledge helpful discussions with many of my colleagues in the UC Davis Department of Ophthalmology and the UC Davis Center for Vision Science-Robert Zawadzki and Jack Werner in particular. The two phase-sensitive AO-OCT efforts in my labscanning and full-field-were led by my postdocs Mehdi Azimipour and Denise Valente, who provided the data used to create (Figure 3) Kari Vienola and Susan Garcia assisted with many of the experiments as well. I would like to thank the National Eye Institute (R00-EY-026068) and the UC Davis Department of Ophthalmology for funding. Funding: National Eye Institute (NEI): R00-EY-026068.

\section{Footnote}

Provenance and Peer Review: This article was commissioned by the Guest Editor (Susanna S. Park) for the series "Novel Tools and Therapies for Ocular Regeneration" published in Annals of Translational Medicine. Available at https://dx.doi. org/10.21037/atm-20-6440

Peer Review File: Available at https://dx.doi.org/10.21037/ atm-20-6440

Conflicts of Interest: The author has completed the ICMJE uniform disclosure form (available at https://dx.doi. org/10.21037/atm-20-6440). The series "Novel Tools and Therapies for Ocular Regeneration" was commissioned by the editorial office without any funding or sponsorship. Dr. RSJ was the recipient of an NIH K99/R00 award and serve on the Optical Society of America's Fall Vision Meeting Planning Committee, as the chair-elect. The author has no other conflicts of interest to declare.

Ethical Statement: The author is accountable for all aspects of the work in ensuring that questions related to the accuracy or integrity of any part of the work are appropriately investigated and resolved.

Open Access Statement: This is an Open Access article distributed in accordance with the Creative Commons Attribution-NonCommercial-NoDerivs 4.0 International License (CC BY-NC-ND 4.0), which permits the noncommercial replication and distribution of the article with 
the strict proviso that no changes or edits are made and the original work is properly cited (including links to both the formal publication through the relevant DOI and the license). See: https://creativecommons.org/licenses/by-nc-nd/4.0/.

\section{References}

1. Rushton WA. Pigments and signals in colour vision. J Physiol 1972;220:1P-P.

2. Rohrschneider K, Bültmann S, Springer C. Use of fundus perimetry (microperimetry) to quantify macular sensitivity. Prog Retin Eye Res 2008;27:536-48.

3. Biersdorf WR. The clinical utility of the foveal electroretinogram: a review. Doc Ophthalmol 1989;73:313-25.

4. Hull BM, Thompson DA. A review of the clinical applications of the pattern electroretinogram. Ophthalmic Physiol Opt 1989;9:143-52.

5. Lai TY, Chan WM, Lai RY, et al. The clinical applications of multifocal electroretinography: a systematic review. Surv Ophthalmol 2007;52:61-96.

6. Tuten WS, Tiruveedhula P, Roorda A. Adaptive optics scanning laser ophthalmoscope-based microperimetry. Optom Vis Sci 2012;89:563-74.

7. Wang Q, Tuten WS, Lujan BJ, et al. Adaptive optics microperimetry and OCT images show preserved function and recovery of cone visibility in macular telangiectasia type 2 retinal lesions. Invest Ophthalmol Vis Sci 2015;56:778-86.

8. Huang D, Swanson EA, Lin CP, et al. Optical coherence tomography. Science 1991;254:1178-81.

9. Drexler W, Morgner U, Kärtner FX, et al. In vivo ultrahigh-resolution optical coherence tomography. Opt Lett 1999;24:1221-3.

10. Drexler W, Morgner U, Ghanta RK, et al. Ultrahighresolution ophthalmic optical coherence tomography. Nat Med 2001;7:502-7.

11. Wojtkowski M, Leitgeb R, Kowalczyk A, et al. In vivo human retinal imaging by Fourier domain optical coherence tomography. J Biomed Opt 2002;7:457-63.

12. de Boer JF, Cense B, Park BH, et al. Improved signal-tonoise ratio in spectral-domain compared with time-domain optical coherence tomography. Opt Lett 2003;28:2067-9.

13. Leitgeb R, Hitzenberger C, Fercher A. Performance of fourier domain vs. time domain optical coherence tomography. Opt Express 2003;11:889-94.

14. Choma M, Sarunic M, Yang C, et al. Sensitivity advantage of swept source and Fourier domain optical coherence tomography. Opt Express 2003;11:2183-9.

15. Liang J, Williams DR, Miller DT. Supernormal vision and high-resolution retinal imaging through adaptive optics. J Opt Soc Am A Opt Image Sci Vis 1997;14:2884-92.

16. Zhang Y, Rha J, Jonnal R, et al. Adaptive optics parallel spectral domain optical coherence tomography for imaging the living retina. Opt Express 2005;13:4792-811.

17. Zawadzki RJ, Jones SM, Olivier SS, et al. Adaptiveoptics optical coherence tomography for high-resolution and high-speed 3D retinal in vivo imaging. Opt Express 2005;13:8532-46.

18. Zhang Y, Cense B, Rha J, et al. High-speed volumetric imaging of cone photoreceptors with adaptive optics spectral-domain optical coherence tomography. Opt Express 2006;14:4380-94.

19. Jonnal RS, Kocaoglu OP, Zawadzki RJ, et al. A Review of Adaptive Optics Optical Coherence Tomography: Technical Advances, Scientific Applications, and the Future. Invest Ophthalmol Vis Sci 2016;57:OCT51-68.

20. Pircher M, Zawadzki RJ. Review of adaptive optics OCT (AO-OCT): principles and applications for retinal imaging Invited. Biomed Opt Express 2017;8:2536-62.

21. Miller DT, Kurokawa K. Cellular-Scale Imaging of Transparent Retinal Structures and Processes Using Adaptive Optics Optical Coherence Tomography. Annu Rev Vis Sci 2020;6:115-48.

22. Grinvald A, Bonhoeffer T, Vanzetta I, et al. Highresolution functional optical imaging: from the neocortex to the eye. Ophthalmol Clin North Am 2004;17:53-67.

23. Mulligan JB, MacLeod DIA, Statler IC. In search of an optoretinogram. NASA Technical Reports, 1994.

24. Sabesan R, Pandiyan VP, Maloney-Bertelli A, et al. Adaptive optics line-field OCT for high-speed imaging of retinal structure and function. Invest Ophthalmol Vis Sci 2019;60:1780.

25. Spaide RF, Curcio CA. Anatomical correlates to the bands seen in the outer retina by optical coherence tomography: literature review and model. Retina 2011;31:1609-19.

26. Jonnal RS, Kocaoglu OP, Zawadzki RJ, et al. The cellular origins of the outer retinal bands in optical coherence tomography images. Invest Ophthalmol Vis Sci 2014;55:7904-18.

27. Spaide RF. Outer Retinal Bands. Invest Ophthalmol Vis Sci 2015;56:2505-6.

28. Jonnal RS, Kocaoglu OP, Zawadzki RJ, et al. Author Response: Outer Retinal Bands. Invest Ophthalmol Vis Sci 2015;56:2507-10.

29. Jonnal RS, Gorczynska I, Migacz JV, et al. The Properties 
of Outer Retinal Band Three Investigated With Adaptive-Optics Optical Coherence Tomography. Invest Ophthalmol Vis Sci 2017;58:4559-68.

30. Schmitt FO, Schmitt OH. Partial excitation and variable conduction in the squid giant axon. J Physiol 1940;98:26-46.

31. Hill DK, Keynes RD. Opacity changes in stimulated nerve. J Physiol 1949;108:278-81.

32. Hill DK. The effect of stimulation on the opacity of a crustacean nerve trunk and its relation to fibre diameter. J Physiol 1950;111:283-303.

33. Hill DK. The volume change resulting from stimulation of a giant nerve fibre. J Physiol 1950;111:304-27.

34. Cohen LB, Keynes RD. Changes in light scattering associated with the action potential in crab nerves. J Physiol 1971;212:259-75.

35. Cohen LB, Keynes RD, Landowne D. Changes in light scattering that accompany the action potential in squid giant axons: potential-dependent components. J Physiol 1972;224:701-25.

36. Cohen LB. Changes in neuron structure during action potential propagation and synaptic transmission. Physiol Rev 1973;53:373-418.

37. Ross WN, Salzberg BM, Cohen LB, et al. Changes in absorption, fluorescence, dichroism, and Birefringence in stained giant axons: optical measurement of membrane potential. J Membr Biol 1977;33:141-83.

38. Hill BC, Schubert ED, Nokes MA, et al. Laser interferometer measurement of changes in crayfish axon diameter concurrent with action potential. Science 1977;196:426-8.

39. Mosbacher J, Langer M, Hörber JK, et al. Voltagedependent membrane displacements measured by atomic force microscopy. J Gen Physiol 1998;111:65-74.

40. Yao XC, Rector DM, George JS. Optical lever recording of displacements from activated lobster nerve bundles and Nitella internodes. Appl Opt 2003;42:2972-8.

41. Lazebnik M, Marks DL, Potgieter K, et al. Functional optical coherence tomography for detecting neural activity through scattering changes. Opt Lett 2003;28:1218-20.

42. Lazebnik M, Marks DL, Potgieter K, et al. Functional optical coherence tomography of stimulated and spontaneous scattering changes in neural tissue. Proceedings of SPIE 2004;5316:107-112.

43. Akkin T, Davé D, Milner T, et al. Detection of neural activity using phase-sensitive optical low-coherence reflectometry. Opt Express 2004;12:2377-86.

44. Akkin T, Joo C, de Boer JF. Depth-resolved measurement of transient structural changes during action potential propagation. Biophys J 2007;93:1347-53.

45. Korenbrot JI, Cone RA. Dark ionic flux and the effects of light in isolated rod outer segments. J Gen Physiol 1972;60:20-45.

46. Bownds D, Brodie AE. Light-sensitive swelling of isolated frog rod outer segments as an in vitro assay for visual transduction and dark adaptation. J Gen Physiol $1975 ; 66: 407-25$.

47. Corless JM. Lamellar structure of bleached and unbleached rod photoreceptor membranes. Nature 1972;237:229-31.

48. Chabre M, Cavaggioni A. Light induced changes in ionic flux in the retinal rod. Nat New Biol 1973;244:118-20.

49. Chabre M, Cavaggioni A. X-ray diffraction studies of retinal rods. II. Light effect on the osmotic properties. Biochim Biophys Acta 1975;382:336-43.

50. McConnell DG. Relationship of the light-induced proton uptake in bovine retinal outer segment fragments to tritoninduced membrane disruption and to volume changes. J Biol Chem 1975;250:1898-906.

51. Hofmann KP, Uhl R, Hoffmann W, et al. Measurements on fast light-induced light-scattering and -absorption changes in outer segments of vertebrate light sensitive rod cells. Biophys Struct Mech 1976;2:61-77.

52. Uhl R, Hofmann KP, Kreutz W. Measurement of fast light-induced disc shrinkage within bovine rod outer segments by means of a light-scattering transient. Biochim Biophys Acta 1977;469:113-22.

53. Harary HH, Brown JE, Pinto LH. Rapid light-induced changes in near infrared transmission of rods in Bufo marinus. Science 1978;202:1083-5.

54. Kaplan MW, Liebman PA. Slow bleach-induced birefringence changes in rod outer segments. J Physiol 1977;265:657-72.

55. Aguirre G, Andrews L. Nomarski evaluation of rod outer segment renewal in a hereditary retinal degeneration. Comparison with autoradiographic evaluation. Invest Ophthalmol Vis Sci 1987;28:1049-58.

56. Kühn H, Bennett N, Michel-Villaz M, et al. Interactions between photoexcited rhodopsin and GTP-binding protein: kinetic and stoichiometric analyses from light-scattering changes. Proc Natl Acad Sci U S A 1981;78:6873-7.

57. Hofmann KP, Schleicher A, Emeis D, et al. Light-induced axial and radial shrinkage effects and changes of the refractive index in isolated bovine rod outer segments and disc vesicles: physical analysis of near-infrared scattering changes. Biophys Struct Mech 1981;8:67-93. 
58. Kühn H, Chabre M. Light-dependent interactions between rhodopsin and photoreceptor enzymes. Biophys Struct Mech 1983;9:231-4.

59. Kahlert M, Hofmann KP. Reaction rate and collisional efficiency of the rhodopsin-transducin system in intact retinal rods. Biophys J 1991;59:375-86.

60. Vuong TM, Chabre M, Stryer L. Millisecond activation of transducin in the cyclic nucleotide cascade of vision. Nature 1984;311:659-61.

61. Vuong TM, Pfister C, Worcester DL, et al. The transducin cascade is involved in the light-induced structural changes observed by neutron diffraction on retinal rod outer segments. Biophys J 1987;52:587-94.

62. Kamps KM, Reichert J, Hofmann KP. Light-induced activation of the rod phosphodiesterase leads to a rapid transient increase of near-infrared light scattering. FEBS Lett 1985;188:15-20.

63. Kamps KM, Hofmann KP. ATP can promote activation and deactivation of the rod cGMP-phosphodiesterase. Kinetic light scattering on intact rod outer segments. FEBS Lett 1986;208:241-7.

64. Pepperberg DR, Kahlert M, Krause A, et al. Photic modulation of a highly sensitive, nearinfrared light-scattering signal recorded from intact retinal photoreceptors. Proc Natl Acad Sci U S A 1988;85:5531-5.

65. Arshavsky VY, Lamb TD, Pugh EN Jr. G proteins and phototransduction. Annu Rev Physiol 2002;64:153-87.

66. Hunter JJ, Merigan WH, Schallek JB. Imaging Retinal Activity in the Living Eye. Annu Rev Vis Sci 2019;5:15-45.

67. Yao X, Kim TH. Fast intrinsic optical signal correlates with activation phase of phototransduction in retinal photoreceptors. Exp Biol Med (Maywood) 2020;245:1087-95.

68. Sabesan R, Hofer H, Roorda A. Characterizing the Human Cone Photoreceptor Mosaic via Dynamic Photopigment Densitometry. PLoS One 2015;10:e0144891.

69. Jonnal RS, Besecker JR, Derby JC, et al. Imaging outer segment renewal in living human cone photoreceptors. Opt Express 2010;18:5257-70.

70. Kocaoglu OP, Liu Z, Zhang F, et al. Photoreceptor disc shedding in the living human eye. Biomed Opt Express 2016;7:4554-68.

71. Izhaky D, Nelson DA, Burgansky-Eliash Z, et al. Functional imaging using the retinal function imager: direct imaging of blood velocity, achieving fluorescein angiography-like images without any contrast agent, qualitative oximetry, and functional metabolic signals. Jpn
J Ophthalmol 2009;53:345-51.

72. Son T, Wang B, Thapa D, et al. Optical coherence tomography angiography of stimulus evoked hemodynamic responses in individual retinal layers. Biomed Opt Express 2016;7:3151-62.

73. Pallikaris A, Williams DR, Hofer H. The reflectance of single cones in the living human eye. Invest Ophthalmol Vis Sci 2003;44:4580-92.

74. Cooper RF, Dubis AM, Pavaskar A, et al. Spatial and temporal variation of rod photoreceptor reflectance in the human retina. Biomed Opt Express 2011;2:2577-89.

75. DeLint PJ, Berendschot TT, van de Kraats J, et al. Slow optical changes in human photoreceptors induced by light. Invest Ophthalmol Vis Sci 2000;41:282-9.

76. Tsunoda K, Oguchi Y, Hanazono G, et al. Mapping cone- and rod-induced retinal responsiveness in macaque retina by optical imaging. Invest Ophthalmol Vis Sci 2004;45:3820-6.

77. Abràmoff MD, Kwon YH, Ts'o D, et al. Visual stimulusinduced changes in human near-infrared fundus reflectance. Invest Ophthalmol Vis Sci 2006;47:715-21.

78. Roorda A, Romero-Borja F, Donnelly Iii W, et al. Adaptive optics scanning laser ophthalmoscopy. Opt Express 2002;10:405-12.

79. Jonnal RS, Rha J, Zhang Y, et al. In vivo functional imaging of human cone photoreceptors. Opt Express 2007;15:16141-60.

80. Rha J, Schroeder B, Godara P, et al. Variable optical activation of human cone photoreceptors visualized using a short coherence light source. Opt Lett 2009;34:3782-4.

81. Bedggood P, Metha A. Variability in bleach kinetics and amount of photopigment between individual foveal cones. Invest Ophthalmol Vis Sci 2012;53:3673-81.

82. Cooper RF, Tuten WS, Dubra A, et al. Non-invasive assessment of human cone photoreceptor function. Biomed Opt Express 2017;8:5098-112.

83. Grieve K, Roorda A. Intrinsic signals from human cone photoreceptors. Invest Ophthalmol Vis Sci 2008;49:713-9.

84. Yao XC, Yamauchi A, Perry B, et al. Rapid optical coherence tomography and recording functional scattering changes from activated frog retina. Appl Opt 2005;44:2019-23.

85. Bizheva K, Pflug R, Hermann B, et al. Optophysiology: depth-resolved probing of retinal physiology with functional ultrahigh-resolution optical coherence tomography. Proc Natl Acad Sci U S A 2006;103:5066-71.

86. Yamauchi Y, Agawa T, Tsukahara R, et al. Correlation between high-resolution optical coherence tomography 
(OCT) images and histopathology in an iodoacetic acidinduced model of retinal degeneration in rabbits. Br J Ophthalmol 2011;95:1157-60.

87. Muraoka Y, Ikeda HO, Nakano N, et al. Real-time imaging of rabbit retina with retinal degeneration by using spectral-domain optical coherence tomography. PLoS One 2012;7:e36135.

88. Srinivasan VJ, Wojtkowski M, Fujimoto JG, et al. In vivo measurement of retinal physiology with high-speed ultrahigh-resolution optical coherence tomography. Opt Lett 2006;31:2308-10.

89. Srinivasan VJ, Chen Y, Duker JS, et al. In vivo functional imaging of intrinsic scattering changes in the human retina with high-speed ultrahigh resolution OCT. Opt Express 2009; 17:3861-77.

90. Schmoll T, Kolbitsch C, Leitgeb RA. In vivo functional retinal optical coherence tomography. J Biomed Opt 2010;15:041513.

91. Berkowitz BA. Oxidative stress measured in vivo without an exogenous contrast agent using QUEST MRI. J Magn Reson 2018;291:94-100.

92. Bissig D, Zhou CG, Le V, et al. Optical coherence tomography reveals light-dependent retinal responses in Alzheimer's disease. Neuroimage 2020;219:117022.

93. Abràmoff MD, Mullins RF, Lee K, et al. Human photoreceptor outer segments shorten during light adaptation. Invest Ophthalmol Vis Sci 2013;54:3721-8.

94. Zhang P, Zawadzki RJ, Goswami M, et al. In vivo optophysiology reveals that $\mathrm{G}$-protein activation triggers osmotic swelling and increased light scattering of rod photoreceptors. Proc Natl Acad Sci U S A 2017;114:E2937-46.

95. Zhang P, Goswami M, Zawadzki RJ, et al. The Photosensitivity of Rhodopsin Bleaching and LightInduced Increases of Fundus Reflectance in Mice Measured In Vivo With Scanning Laser Ophthalmoscopy. Invest Ophthalmol Vis Sci 2016;57:3650-64.

96. Lu CD, Lee B, Schottenhamml J, et al. Photoreceptor Layer Thickness Changes During Dark Adaptation Observed With Ultrahigh-Resolution Optical Coherence Tomography. Invest Ophthalmol Vis Sci 2017;58:4632-43.

97. Choma MA, Ellerbee AK, Yang C, et al. Spectral-domain phase microscopy. Opt Lett 2005;30:1162-4.

98. Jonnal RS, Kocaoglu OP, Wang Q, et al. Phase-sensitive imaging of the outer retina using optical coherence tomography and adaptive optics. Biomed Opt Express 2012;3:104-24.

99. Kocaoglu OP, Lee S, Jonnal RS, et al. Imaging cone photoreceptors in three dimensions and in time using ultrahigh resolution optical coherence tomography with adaptive optics. Biomed Opt Express 2011;2:748-63.

100. Kocaoglu OP, Turner TL, Liu Z, et al. Adaptive optics optical coherence tomography at $1 \mathrm{MHz}$. Biomed Opt Express 2014;5:4186-200.

101. Klein T, Wieser W, Eigenwillig CM, et al. Megahertz OCT for ultrawide-field retinal imaging with a 1050 $\mathrm{nm}$ Fourier domain mode-locked laser. Opt Express 2011;19:3044-62.

102. Migacz JV, Gorczynska I, Azimipour M, et al. Megahertzrate optical coherence tomography angiography improves the contrast of the choriocapillaris and choroid in human retinal imaging. Biomed Opt Express 2018;10:50-65.

103. Bonin T, Franke G, Hagen-Eggert M, et al. In vivo Fourier-domain full-field OCT of the human retina with 1.5 million A-lines/s. Opt Lett 2010;35:3432-4.

104.Hillmann D, Lührs C, Bonin T, et al. Holoscopy-holographic optical coherence tomography. Opt Lett 2011;36:2390-2.

105.Hillmann D, Spahr H, Sudkamp H, et al. Off-axis reference beam for full-field swept-source OCT and holoscopy. Opt Express 2017;25:27770-84.

106. Borycki D, Auksorius E, Węgrzyn P, et al. Computational aberration correction in spatiotemporal optical coherence (STOC) imaging. Opt Lett 2020;45:1293-6.

107.Auksorius E, Borycki D, Wojtkowski M. Multimode fiber enables control of spatial coherence in Fourier-domain full-field optical coherence tomography for in vivo corneal imaging. Opt Lett 2021;46:1413-6.

108. Hillmann D, Spahr H, Hain C, et al. Aberration-free volumetric high-speed imaging of in vivo retina. Sci Rep 2016;6:35209.

109. Adie SG, Graf BW, Ahmad A, et al. Computational adaptive optics for broadband optical interferometric tomography of biological tissue. Proc Natl Acad Sci U S A 2012;109:7175-80.

110. South FA, Kurokawa K, Liu Z, et al. Combined hardware and computational optical wavefront correction. Biomed Opt Express 2018;9:2562-74.

111.Hillmann D, Spahr H, Pfäffle C, et al. In vivo optical imaging of physiological responses to photostimulation in human photoreceptors. Proc Natl Acad Sci U S A 2016;113:13138-43.

112.Pfäffle C, Spahr H, Kutzner L, et al. Simultaneous functional imaging of neuronal and photoreceptor layers in living human retina. Opt Lett 2019;44:5671-4.

113.Azimipour M, Migacz JV, Zawadzki RJ, et al. Functional 
retinal imaging using adaptive optics swept-source OCT at 1.6 MHz. Optica 2019;6:300-3.

114.Zhang F, Kurokawa K, Lassoued A, et al. Cone photoreceptor classification in the living human eye from photostimulation-induced phase dynamics. Proc Natl Acad Sci U S A 2019;116:7951-6.

115. Curcio CA, Owsley C, Jackson GR. Spare the rods, save the cones in aging and age-related maculopathy. Invest Ophthalmol Vis Sci 2000;41:2015-8.

116. Neely D, Zarubina AV, Clark ME, et al. Association between visual function and subretinal drusenoid deposits in normal and early age-related macular degeneration eyes. Retina 2017;37:1329-36.

117. Curcio CA, Owsley C. Rod-Mediated Dark Adaptation as a Suitable Outcome for Early and Intermediate Age-Related Macular Degeneration. Ophthalmology 2019;126:866-7.

118. Dubra A, Sulai Y, Norris JL, et al. Noninvasive imaging of the human rod photoreceptor mosaic using a confocal adaptive optics scanning ophthalmoscope. Biomed Opt Express 2011;2:1864-76.

119.Azimipour M, Jonnal RS, Werner JS, et al. Coextensive synchronized SLO-OCT with adaptive optics for human retinal imaging. Opt Lett 2019;44:4219-22.

120.Azimipour M, Valente D, Vienola KV, et al. Optoretinogram: optical measurement of human cone and rod photoreceptor responses to light. Opt Lett 2020;45:4658-61.

121. Adie SG, Shemonski ND, Graf BW, et al. Guide-starbased computational adaptive optics for broadband interferometric tomography. Appl Phys Lett 2012;101:221117.

122. Kumar A, Drexler W, Leitgeb RA. Subaperture correlation based digital adaptive optics for full field optical coherence tomography. Opt Express 2013;21:10850-66.

123. Ginner L, Kumar A, Fechtig D, et al. Noniterative digital aberration correction for cellular resolution retinal optical coherence tomography in vivo. Optica 2017;4:924-31.

124. Kumar A, Georgiev S, Salas M, et al. Digital adaptive optics based on digital lateral shearing of the computed pupil field for point scanning retinal swept source OCT. Biomed Opt Express 2021;12:1577-92.

125.Pandiyan VP, Jiang X, Maloney-Bertelli A, et al. Highspeed adaptive optics line-scan OCT for cellular-resolution optoretinography. Biomed Opt Express 2020;11:5274-96.

126.Valente D, Vienola KV, Zawadzki RJ, et al. Kilohertz retinal FF-SS-OCT and flood imaging with hardware-based adaptive optics. Biomed Opt Express
2020;11:5995-6011.

127.Pandiyan VP, Maloney-Bertelli A, Kuchenbecker JA, et al. The optoretinogram reveals the primary steps of phototransduction in the living human eye. Sci Adv 2020;6:eabc1124.

128. Boyle KC, Chen ZC, Ling T, et al. Mechanisms of LightInduced Deformations in Photoreceptors. Biophys J 2020;119:1481-8.

129. Cone RA. EARLY RECEPTOR POTENTIAL OF THE VERTEBRATE RETINA. Nature 1964;204:736-9.

130. Brown KT. The eclectroretinogram: its components and their origins. Vision Res 1968;8:633-77.

131.Sandberg MA, Pawlyk BS, Dan J, et al. Rod and cone function in the Nougaret form of stationary night blindness. Arch Ophthalmol 1998;116:867-72.

132. Muradov KG, Artemyev NO. Loss of the effector function in a transducin-alpha mutant associated with Nougaret night blindness. J Biol Chem 2000;275:6969-74.

133. Moussaif M, Rubin WW, Kerov V, et al. Phototransduction in a transgenic mouse model of Nougaret night blindness. J Neurosci 2006;26:6863-72.

134. Michaelides M, Aligianis IA, Holder GE, et al. Cone dystrophy phenotype associated with a frameshift mutation (M280fsX291) in the alpha-subunit of cone specific transducin (GNAT2). Br J Ophthalmol 2003;87:1317-20.

135.Pircher M, Kroisamer JS, Felberer F, et al. Temporal changes of human cone photoreceptors observed in vivo with SLO/OCT. Biomed Opt Express 2010;2:100-12.

136.Arshavsky VY, Burns ME. Current understanding of signal amplification in phototransduction. Cell Logist 2014;4:e29390.

137. Walther G, Hellner KA. Early receptor potential recordings for clinical routine. Doc Ophthalmol 1986;62:31-9.

138.Gabriele ML, Wollstein G, Ishikawa H, et al. Three dimensional optical coherence tomography imaging: advantages and advances. Prog Retin Eye Res 2010;29:556-79.

139. Putnam NM, Hammer DX, Zhang Y, et al. Modeling the foveal cone mosaic imaged with adaptive optics scanning laser ophthalmoscopy. Opt Express 2010;18:24902-16.

140. Fingler J, Schwartz D, Yang C, et al. Mobility and transverse flow visualization using phase variance contrast with spectral domain optical coherence tomography. Opt Express 2007;15:12636-53.

141. Makita S, Kurokawa K, Hong YJ, et al. Noise-immune complex correlation for optical coherence angiography based on standard and Jones matrix optical coherence 
tomography. Biomed Opt Express 2016;7:1525-48.

142.Spahr H, Pfäffle C, Burhan S, et al. Phase-sensitive interferometry of decorrelated speckle patterns. Sci Rep 2019;9:11748.

143.Badon A, Barolle V, Irsch K, et al. Distortion matrix concept for deep optical imaging in scattering media. Sci Adv 2020;6:eaay7170.

144.Lu Y, Kim TH, Yao X. Comparative study of wildtype and rd10 mice reveals transient intrinsic optical signal response before phosphodiesterase activation

Cite this article as: Jonnal RS. Toward a clinical optoretinogram: a review of noninvasive, optical tests of retinal neural function. Ann Transl Med 2021;9(15):1270. doi: 10.21037/ atm-20-6440 in retinal photoreceptors. Exp Biol Med (Maywood) 2020;245:360-7.

145.Azimipour M, Valente D, Vienola KV, et al. Investigating the functional response of human cones and rods with a combined adaptive optics SLO-OCT system. SPIE Proceedings 2020;11218.

146.Lassoued A, Zhang F, Kurokawa K, et al. Measuring dysfunction of cone photoreceptors in retinitis pigmentosa with phase-sensitive AO-OCT. SPIE Proceedings $2020 ; 11218$. 